\title{
Discours
}

Revue de linguistique, psycholinguistique et

informatique. A journal of linguistics, psycholinguistics and computational linguistics

$18 \mid 2016$

Varia

\section{Acquisition des pronoms objets en français langue seconde}

Céline Pozniak et Barbara Hemforth

(2) OpenEdition

Journals

Édition électronique

URL : http://journals.openedition.org/discours/9151

DOI : 10.4000/discours. 9151

ISSN : 1963-1723

Éditeur :

Laboratoire LATTICE, Presses universitaires de Caen

Référence électronique

Céline Pozniak et Barbara Hemforth, «Acquisition des pronoms objets en français lanque seconde », Discours [En ligne], 18 | 2016, mis en ligne le 16 septembre 2016, consulté le 01 mai 2019. URL : http:// journals.openedition.org/discours/9151 ; DOI : 10.4000/discours.9151 

Revue de linguistique, psycholinguistique et informatique

\section{Acquisition des pronoms objets en français langue seconde}

Céline Pozniak

Laboratoire de linguistique formelle

CNRS - UMR 7110

Barbara Hemforth

Laboratoire de linguistique formelle

CNRS - UMR 7110

Céline Pozniak, Barbara Hemforth, « Acquisition des pronoms objets en français langue seconde», Discours [En ligne], 18 | 2016, mis en ligne le 16 septembre 2016.

URL: http://discours.revues.org/9151

Titre du numéro: Varia

Coordination: Saveria Colonna \& Sarah Schimke 



\title{
Acquisition des pronoms objets en français langue seconde
}

\author{
Céline Pozniak \\ Laboratoire de linguistique formelle \\ CNRS - UMR 7110 \\ Barbara Hemforth \\ Laboratoire de linguistique formelle \\ CNRS - UMR 7110
}

L'acquisition des pronoms objets en français langue seconde (L2) est une réelle difficulté pour tous les apprenants, quelle que soit leur langue maternelle (L1). En s'inspirant de l'étude de Grüter et Crago (2012), nous avons réalisé une expérience de production ainsi qu'un test de mémoire de travail avec deux groupes d'apprenants dont la L1 est respectivement l'allemand et le chinois mandarin. Un groupe de locuteurs natifs du français a été inclus également en tant que groupe de contrôle. Notre expérience permet d'approfondir la recherche sur les pronoms objets notamment en regardant leur maîtrise par les apprenants dans différents contextes et selon différents types de verbes. Les résultats montrent le même pattern pour les deux groupes d'apprenants. Également, on constate que les pronoms objets semblent être mieux maîtrisés dans les contextes appropriés que les réfléchis, que ce soit pour le groupe allemand ou le groupe chinois, ce qui indiquerait une difficulté spécifique de cette forme. Nous envisagerons finalement le fait que le facteur déterminant ne serait pas uniquement la langue maternelle ou la compétence en L2 mais également la mémoire de travail.

Mots clés: acquisition, bilinguisme, mémoire de travail, production, clitique objet, français

Acquisition of object pronouns in L2 French is always a source of problems for every learne regardless of their L1. Based on a study from Grüter and Crago (2012), we ran an elicited production experiment as well as a Reading Span task with two groups of learners whose L1 is German and Mandarin Chinese. A group of French native speakers was added as a control group. We looked at the acquisition of object pronouns in different contexts and depending on the verb type. Our results show that the two groups of learners have a similar pattern of production. Furthermore, reflexive pronouns seem to be the most difficult type of pronouns to master for both groups of Mandarin Chinese and German. This indicates that reflexives are different from other object pronouns and more difficult to master. Our findings suggest that influence of the L1 and level in L2 French are not the major factors for explaining Second Language Acquisition of object pronouns, but that Working Memory does play a role as well.

Keywords: acquisition, bilingualism, working memory, production, object pronoun, French

\section{Introduction}

L'apprentissage du français en langue seconde (dorénavant L2), à savoir toute langue autre que la langue maternelle $(\mathrm{LI})$, pose la question des difficultés et des limites de l'acquisition de certaines catégories fonctionnelles, pouvant mener, pour quelques-unes, à une fossilisation de l'interlangue de l'apprenant (Selinker, 1972). 
Grüter et Crago (20I2) constatent à ce sujet la difficulté de l'acquisition en L2 des pronoms clitiques objets ${ }^{1}$, considérés comme l'une des dernières catégories fonctionnelles à être acquise correctement. Dans cette étude, nous avons analysé la production en $\mathrm{L}_{2}$ des pronoms objets directs mais aussi des réfléchis afin d'établir une comparaison entre ces deux structures considérées toutes les deux comme des clitiques. Trois grands facteurs qui pourraient avoir un impact sur leur acquisition seront considérés: l'influence de la Li qui comporterait ou non cette catégorie, des difficultés de mémoire de travail, ou un problème d'interface entre les structures linguistiques et d'autres domaines cognitifs (Sorace, 20II).

Dans la lignée de Grüter et Crago (2012), nous avons réalisé une expérience de production en français avec des locuteurs natifs du mandarin, des locuteurs natifs de l'allemand et des locuteurs natifs du français en tant que groupe de contrôle. La comparaison de trois groupes différents de locuteurs ainsi que la manipulation des clitiques selon le type de verbes (qui, selon le contexte, peuvent prendre différents types de clitiques ou ne pas en prendre du tout) permettent d'enrichir les résultats de Grüter et Crago (2012) et d'aller plus loin pour comprendre les facteurs impliqués dans l'acquisition des clitiques objets en français L2, notamment en ce qui concerne l'influence de la LI.

Nous développerons dans cette introduction la nature de la catégorie fonctionnelle étudiée, son acquisition en $\mathrm{L}_{1}$ et en $\mathrm{L}_{2}$, ce qui nous amènera aux différentes hypothèses proposées relatives à ce sujet. La deuxième partie détaillera l'expérience élaborée concernant la production des clitiques objets et réfléchis. Nous discuterons les résultats et donnerons notre conclusion là-dessus dans la troisième partie.

\subsection{Les clitiques objets: propriétés \\ 1.1.1. Le français}

$4 \quad$ Les clitiques (ou pronoms faibles) remplacent un syntagme et renvoient à ce qu'il désigne (animé ou inanimé). Ils n’ont pas de référence intrinsèque. Ils se classent dans les catégories fonctionnelles, ce qui signifie qu'ils n'ont pas de contenu descriptif et constituent seulement un rôle grammatical (Riegel et al., 2009). Ils s'appuient phonologiquement sur un hôte pour former un mot prosodique. Ils se placent en français avant l'hôte, que ce soit pour une forme finie ou non finie, sauf à l'impératif (exemple [I]). Les exemples suivants sont inspirés de ceux de Miller et Monachesi (2003).

[I] a. Anna le regarde.

b. Anna ne fait que le regarder.

c. Regarde-le.

1. Il est vrai que l'appellation «clitique» est débattue, notamment pour les pronoms en français (voir, entre autres, Miller, 1992). Même si l'argumentation en faveur des affixes pour le français est très convaincante, nous resterons avec le terme de clitiques afin d'être plus proches de la littérature en acquisition des $\mathrm{Lr}_{\mathrm{r}}$ et L2 qui utilise ce terme dans les expériences. 
Les clitiques se distinguent des autres pronoms, dits forts, en ce sens qu'ils ont, comme nous allons le montrer, des propriétés bien spécifiques (voir, entre autres, Miller et Monachesi, 2003; Prévost, 2009; Riegel et al., 2009).

Ils n'occupent pas la même position syntaxique que le syntagme nominal (SN) ou le syntagme prépositionnel (SP) pleins qu'ils remplacent (exemple [2]). Ils doivent être contigus au verbe, et donc ne peuvent pas en être séparés si ce n'est par un autre clitique ou par la négation, et ils ne peuvent pas apparaitre de manière isolée (exemple [3]). La coordination est impossible avec des clitiques en français (exemple [4]). À nouveau, les clitiques diffèrent des syntagmes pleins en ce qui concerne le positionnement syntaxique. Ils ne peuvent pas être modifiés (exemple [5]). Ils sont normalement inaccentués et ne peuvent pas porter un accent de contraste (exemple [6]). Enfin, ils ne peuvent pas être résomptifs et apparaître avec le complément d'objet auquel ils réfèrent en français (exemple [7]) ${ }^{2}$.

[2] a. Anna regarde $[\mathrm{le} \mathrm{film}]_{S N}$.

b. ${ }^{*}$ Anna regarde $[\mathrm{le}]_{C L}$.

c. Anna $[\mathrm{le}]_{C L}$ regarde.

[3] a. *Anna le parfois regarde.

b. Anna le regarde parfois.

c. Que regarde Anna? * Le.

[4] a. Anna voit la fille et le garçon.

b. *Anna voit la et le.

c. ${ }^{*}$ Anna voit la fille et je.

[5] * Je les deux vois.

[6] *Je LE vois.

[7] *Anna le ${ }_{i}$ regarde le film.

Pour notre expérience, nous ne retiendrons que les caractéristiques suivantes: la différence de positionnement syntaxique avec le SN/SP plein comme en [2], le rapport avec le verbe (exemple [3]) et la non résomptivité (exemple [7]).

Les clitiques objets à la troisième personne varient en genre et en nombre (exemple [8]). Le clitique objet peut être dit réfléchi et aura des propriétés différentes en ce sens qu'il incarnera le même référent que le sujet, comme en [9]. Sa forme à la troisième personne est invariable quels que soient le genre et le nombre et reste se. Ce clitique est considéré comme étant également parmi les clitiques objets directs.

2. Excepté si l'on a une dislocation à droite avec une virgule après le verbe: Anna le regarde, le film. 
[8] a. Je $\mathrm{la}_{\text {féminin/singulier }}$ vois.

b. Je le $\mathrm{l}_{\text {masculin/singulier }}$ vois.

c. Je les ${ }_{\text {pluriel }}$ vois.

[9] $\mathrm{Il}_{i} \mathrm{se}_{i}$ lave.

\subsubsection{Le chinois mandarin}

Le mandarin est une langue dont la morphologie est peu marquée. De fait, les phrases sont souvent elliptiques et on ne constate pas vraiment de marques d'agent ou de patient. Il faut alors s'appuyer généralement sur le contexte (Xu, 20ro).

Il n'existe pas de clitiques objets en chinois mandarin comme ceux présents en français ${ }^{3}$. Dans ce cas, on peut reprendre le complément d'objet ou bien avoir une construction à objet nul identifié par le contexte (exemple [ıo]).

[Iо] a. Français

Est-ce que tu bois du café? Oui, j'en bois.

b. Mandarin

你喝咖啡吗 Tu-bois-café-part.inter./Est-ce que tu bois du café?

我喝。Je-bois/Je bois

我喝呦啡。Je-bois-café/Je bois café.

喝。Bois/Bois

En ce qui concerne la forme réfléchie, deux formes de pronom sont possibles: 自 $(z i)$ et 自己 $(z i j \tilde{i})$ qui signifient soi, soi-même. Il est possible de les combiner avec d'autres pronoms comme 我 (wǒ, «je»), 他 $(t \bar{a}$, «il»), entre autres. De façon générale, ces pronoms sont employés pour insister sur le «soi» d'un être ou d'un objet (Darobbers et Xiao Planes, 1998). Lorsqu'ils sont employés devant un verbe, il ont une valeur adverbiale.

De fait, il apparaît que le clitique réfléchi se français n'existe pas comme tel en mandarin et que le réfléchi dans cette langue se comporte plus comme le réfléchi en anglais (pour plus d'informations, voir Huang, Li et Li [2009]). Comme en [II], on retrouvera, pour exprimer la forme réfléchie, des groupes de mots (verbe + objet) ou seulement le verbe (Vinet et Zhou, 2003).

[II] a. Français

Je me brosse.

b. Mandarin

我洗漱。Je-brosse/Je brosse.

3. Excepté peut-être la particule suo qui existe spécifiquement dans les relatives objets pour marquer le SN objet (pour plus d'informations, voir Chiu, 1995). 


\subsubsection{L'allemand}

L'allemand ne possède pas non plus de clitiques objets comme cela est constaté en français. Les pronoms allemands remplacent le SN objet dans la même position syntaxique (exemple [I2]), comme en chinois mandarin, excepté si l'effet de longueur et/ou l'effet de structure informationnelle entrent en jeu, mais ils varient en genre et en nombre (exemple [13]). De plus, l'allemand ne permet généralement ${ }^{4}$ pas, contrairement au chinois mandarin, l'objet nul (voir exemple [I4]). Il existe, enfin, la forme réfléchie en allemand pour les pronoms objets, qui remplace le SN objet dans la même position syntaxique et qui diffère du réfléchi français (exemple [I5]).

[I2] a. Ich kaufe das Buch. J'achète le livre.

b. Ich kaufe es. J'achète-le.

[13] a. Ich kaufe sie. J'achète-la

b. Ich kaufe es. J'achète-le

c. Ich kaufe $i b n$. J'achète-le

d. Ich kaufe sie. J'achète-les phriril $_{\text {f }}$

[I4] a. Kaufst du das Buch? - *Ich kaufe. Est-ce que tu achètes le livre? - J'achète.

\section{b. 你买书吗 - 我买}

Est-ce que tu acbètes le livre? - J'achète.

[ıร] $\quad$ Er $_{i}$ wäscht sich. Il se lave.

\subsection{Acquisition des clitiques objets en $\mathrm{L}_{1}$}

L'acquisition en Li est une question intéressante pour faire le lien avec l'acquisition en L2 et mieux comprendre cette dernière, particulièrement par rapport à la possibilité selon laquelle la L2 pourrait s'acquérir comme la Li. Concernant cela, White (2003) propose trois hypothèses. La première hypothèse est que l'acquisition en L2 suivrait celle de la Li avec un accès direct à une "grammaire universelle». Une autre hypothèse

4. Il existe des exceptions avec un objet nul quand le référent est facilement accessible dans le contexte - comme Mach auf(«Ouvre!») pour Macb die Tür auf(«Ouvre la porte»), ou Gib her! («Donne!») pour Gib es her! («Donne-le!») - mais l'omission du pronom objet reste plutôt rare (Štícha, 20I5). 
est que l'acquisition en L2 serait influencée par la Li avec un accès indirect. Dans ce cas, le stade initial dépend de la grammaire de la LI, que ce soit complètement (Schwartz et Sprouse, I994 et 1996) ou partiellement (Vainikka et Young-Scholten, 1994 et 1996). Trois propositions sont exprimées à ce sujet: i) possibilité de refixation des paramètres vers ceux de la L2 menant à une performance proche de celle des locuteurs natifs, ou ii) maintien des paramètres de la LI. L'acquisition en L2 pourrait aussi être considérée comme iii) étant complètement différente de celle de la Li avec des mécanismes spécifiques car les apprenants n'auraient plus accès à une "grammaire universelle». Finalement, une dernière hypothèse est que le niveau en L2 pourrait être semblable à celui en Li grâce à l'entraînement et à l'usage, comme ce que propose le modèle de compétition de Hernandez, Li et MacWhinney (2005). Dans ce modèle, la Li et la L2 sont en compétition, et la Li, plus ancrée chez l'apprenant, domine. Cependant, plus l'apprenant avance dans la L2, plus cette asymétrie entre les deux langues disparait.

Hamann et al. (1996) ont remarqué que les enfants reconnaissent les clitiques très tôt, ceci laissant supposer que les catégories fonctionnelles seraient présentes dans la grammaire de l'enfant à un stade précoce. Vers approximativement l'âge de 2 ans, ils arrivent à différencier un SN plein d'un clitique (Tsedryk et Punko, 2008a). Également, ils produisent les clitiques objets directs plus tard que les clitiques sujets, mais sembleraient les produire plus tôt que les clitiques objets indirects (lui, leur, etc.), selon Tsedryk et Punko (2008a). Avant cette maitrise, Prévost (2009) constate que les enfants vont d'abord remplacer les clitiques objets par des pronoms forts (moi), ou par le pronom démonstratif ça, par des compléments d'objet, ou encore ils vont omettre les clitiques objets ainsi que les compléments d'objet (exemple [i6]).

[16] a. Tu choisis qui? - Je choisis toi.

b. Tu choisis quoi? - Je choisis ça.

c. Tu choisis ce jouet? - Je choisis ce jouet.

d. Tu choisis ce jouet? - Je choisis

Cela étant, lorsque les enfants commencent à produire des clitiques, ces derniers sont presque toujours bien placés et utilisés directement de façon grammaticalement correcte. En outre, les enfants comprennent préalablement mieux la référence des clitiques qu'ils n'arrivent à les produire (pour plus d'informations, voir Hamann et al., 1996; Prévost, 2009). Prévost (2009) fait également remarquer que les réfléchis semblent apparaitre plus tôt que les clitiques objets (entre autres, Jakubowicz et Rigaut, 2000; Van der Velde, 2003).

Cette acquisition tardive s'explique régulièrement par des problèmes de mémoire de travail. Ainsi, Jakubowicz et al. (1998) va proposer «l'hypothèse de complexité computationnelle» (Computational Complexity Hypothesis). Les clitiques objets sont non spécifiés, c'est-à-dire qu'ils ne réfèrent pas de façon intrinsèque à un être animé ou inanimé, contrairement aux noms ou pronoms forts. Cette propriété expliquerait en partie pourquoi les enfants préféreraient ne rien produire et répéter le complément objet (exemple [i7]). 
[17] a. Que fait Papa avec le chien? - *Papa brosse

b. Que fait Papa avec le chien? - Papa brosse le chien.

Une autre hypothèse est celle de «l'interface» (Interface Hypothesis), de Sorace et Filiaci (2006), qui stipule que les catégories en contact avec d'autres domaines

Également, Grüter (2006) propose «l'hypothèse des traits détériorés» (Decayed Feature Hypothesis) qui considère, comme avec Jakubowicz, que l'acquisition tardive des clitiques objets résulte de problèmes de capacités de mémoire de travail. Cette hypothèse s'appuie sur la relation de dépendance entre le clitique et l'élément pro qui se situe dans le complément du syntagme verbal (verb phrase [VP]; Grüter, 2006). Ces deux éléments ne vont pas être fusionnés (ou unifiés) en même temps à une distance considérable (opération «Merge» selon Grüter, 2006). En effet, le clitique va être fusionné après plusieurs opérations computationnelles et notamment après avoir franchi une frontière de catégorie syntaxique (VP). Selon Grüter (2006), au moment de fusionner le clitique, l'élément pro aura perdu quelques traits, ce qui rendra la vérification des traits et la relation entre les éléments difficiles si la mémoire de travail est restreinte. Les enfants ne vont alors pas traiter correctement les dépendances de longue distance, ils vont omettre le complément d'objet et ne vont pas réussir à réaliser le clitique correspondant en raison d'une capacité de mémoire de travail réduite.

De fait, si l'acquisition de la L2 ressemble à celle de la LI, les problèmes constatés en LI, particulièrement liés à la mémoire de travail devraient se retrouver en L2. Si tel n'est pas le cas, alors l'influence de la Li est un autre facteur possible, mais également d'autres effets très différents de la $\mathrm{L}_{\mathrm{I}}$ (voir I.3).

\subsection{Acquisition des clitiques objets en $L_{2}$}

Si l'acquisition en L2 diffère de l'acquisition en LI, l'acquisition des clitiques objets en L2 va présenter des particularités bien distinctes. L'acquisition en L2 de cette catégorie syntaxique n'est effectivement pas systématique. Prévost (2009) note d'ailleurs que les réfléchis apparaissent plus tôt et sont utilisés correctement d'un point de vue syntaxique, même si rien n'affirme que ces éléments soient véritablement compris et analysés par les apprenants en L2. Suivant cette perspective, les différences possibles entre Li et L2 vont être expliquées selon plusieurs hypothèses.

La première hypothèse prendrait en compte l'influence de la Li qui jouerait un rôle considérable lors de l'apprentissage de la L2. D'après cette hypothèse, l'apprenant en L2 va acquérir les catégories fonctionnelles par la Li. Les apprenants en L2 vont alors projeter les structures syntaxiques compatibles avec la grammaire de leur Li. White (1996) constate d'ailleurs que des apprenants enfants avec pour Li l'anglais et pour L2 le français ont des difficultés à produire des clitiques et à comprendre leur coréférence et leur fonctionnement, en partie parce que l'anglais ne possède pas cette catégorie fonctionnelle. Ainsi, ces apprenants traitent les clitiques comme les pronoms forts de leur Li (exemple [I8]).

[r8] Qu'est-ce que tu fais avec le gâteau? - *Je mange le. 
cognitifs vont s'avérer être les plus difficiles à acquérir. L'interface se définit par des structures syntaxiques sensibles à plusieurs conditions de nature différente. Ces différentes conditions doivent être satisfaites afin que les structures soient correctement construites. On distingue les structures en relation avec d'autres domaines cognitifs comme «l'interface syntaxe-pragmatique» (Syntax-Pragmatics) et les autres n'étant pas en relation avec ces derniers comme «l'interface syntaxe-lexique» (Syntax-Lexicon). Une différence est donc établie entre des structures linguistiques qui requièrent des conditions de nature uniquement interne à la grammaire (interface syntaxe-lexique), soit les interfaces internes, et des structures linguistiques qui requièrent une intégration des facteurs contextuels (interface syntaxe-pragmatique), soit les interfaces externes. Selon Sorace et Filiaci (2006), les structures impliquant une interface externe présentent une option résiduelle dans l'acquisition en L2, une option émergente dans l'attrition de la $\mathrm{LI}^{5}$ et une indétermination à long terme en acquisition bilingue. Cependant, les structures langagières impliquant une interface interne sont complètement assimilées en L2, restent stables en Lr et sont assimilées tôt en acquisition bilingue.

Dans un autre cadre, Clahsen et Felser (2006a et b) proposent que l'influence de la Li se limite à certains domaines comme le domaine lexical et le domaine phonologique. Ils considèrent alors que l'acquisition en $\mathrm{L}_{2}$ va principalement relever de stratégies différentes. L'apprenant en L2 va effectivement montrer des performances similaires à celles d'un locuteur natif dans certains domaines linguistiques précis tels que la sémantique et la syntaxe (cette dernière se caractérisant par les relations grammaticales telles que la concordance du genre dans un SN et l'accord sujet-verbe dans les dépendances locales). Il ne va cependant pas recourir aux mêmes stratégies pour les relations syntaxiques dans les dépendances non locales. Pour interpréter une phrase, le locuteur natif va mettre en jeu deux processus, «l'analyse complète de la phrase» (Full Parsing) pour fournir une représentation complète, et «l'analyse en surface de la phrase» (Shallow Parsing) pour se concentrer plus sur l'information sémantique et lexicale de la phrase. D'autres recherches ont d'ailleurs montré le recours régulier à l'analyse en surface par les locuteurs natifs plutôt qu'à l'analyse complète (Ferreira et al., 2002; Ferreira, 2003). S'inspirant de cela, «l'hypothèse de la structure de surface» (Shallow Structure Hypothesis) va partir du principe que l'apprenant en L2 aura principalement recours à «l'analyse de surface» (Shallow Parsing).

La dernière hypothèse concerne la mémoire de travail comme dans l'article de Grüter et Crago (2012) qui proposent une expérience de compréhension («tâche de jugement de valeur de vérité», Truth-Value Judgment Task) et de production élicitée de clitiques objets ainsi que deux tests de mémoire de travail: «l'empan de chiffres inversés» (Backward Digit Recall Span) qui mesurerait la composante exécutive de la mémoire de travail, et «l'empan de répétition de non-mots» (Non-Word Repetition Span) qui refléterait la mémoire de travail verbale. Les participants étaient deux groupes d'enfants chinois et espagnols âgés en moyenne de 8 ans. Les enfants

5. Perte des compétences dans la Li liée à l'âge ou à une immersion totale en L2. 
chinois sont des bilingues tardifs avec pour L2 le français, dont l'âge d'acquisition moyen est de 5 ans. La tâche de production élicitée était composée d'histoires sur une petite fille et son chien avec des images. L'expérimentateur posait une question relative à l'image, qui devait favoriser la production de clitiques objets. La tâche de compréhension se composait d'images et les enfants devaient dire si les phrases énoncées par une marionnette (celle de l'expérimentateur) étaient vraies ou fausses. La tâche de compréhension montre que les enfants rejettent l'interprétation de phrases françaises avec un complément d'objet non réalisé même si cela est acceptable en chinois. Leur interlangue ne permet pas d'accepter le complément d'objet nul et l'influence de la Li ne serait donc pas aussi importante dans le cadre de cette expérience. Ce qui interviendrait alors ici serait plutôt des problèmes de mémoire de travail. Cette hypothèse est confirmée par le fait que les enfants qui omettaient les clitiques n'avaient pas des résultats très élevés dans les tests de mémoire de travail («empan de chiffres inversés», Backward Digit Recall Span). Grüter et Crago (2012) proposent d'expliquer, à partir de leur expérience, les difficultés pour l'acquisition de cette catégorie fonctionnelle en utilisant la "grammaire d'arbres adjoints» (Tree-Adjoining Grammar [TAG]) de Joshi et al. (1975), comme Ferreira (2000) l'a proposé pour la production en psycholinguistique. Selon Grüter et Crago (20I2), les clitiques représentent une catégorie fonctionnelle requérant deux opérations lors de sa réalisation: la substitution et l'adjonction. La substitution représente la combinaison de deux arbres syntaxiques élémentaires par l'insertion d'un des deux dans le nœud périphérique de l'autre. Pour les clitiques, on va ainsi substituer le sujet (ici le dragon) dans le nœud de la projection appropriée (figure I). L'adjonction (Adjoining) consiste en l'insertion d'une structure dans une autre. Pour les clitiques, il va falloir adjoindre le clitique dans le nœud approprié de la projection verbale, puis substituer l'élément pro dans le complément verbal pour satisfaire les arguments (figure 2). L'adjonction étant une opération particulièrement difficile à réaliser, la production de phrases avec des clitiques objets demanderait plus d'efforts cognitifs. Les apprenants effectueraient donc toutes les phases de mise en place du clitique mais n'arriveraient pas à bien réaliser l'étape du déplacement de ce dernier en position préverbale, ce qui conduit alors à des phrases avec omission du complément d'objet. Ceci est alors à mettre en parallèle avec l'hypothèse de complexité computationnelle de Jakubowicz et al. (1998) pour la Li.

L'acquisition des clitiques objets en L2 a déjà suscité de nombreuses études. Grüter et Crago (20I2) entre autres ont analysé la production des clitiques objets directs chez les enfants en L2 et ont observé que les clitiques objets directs français étaient produits plus facilement par des locuteurs dont la Li (dans ce cas, l'espagnol) comporte également des clitiques que par des locuteurs chinois qui n'ont pas de clitiques dans leur Li. Pour l'acquisition en L2 chez les adultes, Tsedryk et Punko (2008a) se sont concentrés sur la différence entre clitiques objets directs et indirects chez des locuteurs natifs de l'anglais en français L2. Ils ont mis en place des tests de jugement de grammaticalité et de traduction sur des phrases écrites avec un groupe de niveau intermédiaire et un groupe de niveau avancé, ainsi qu'un groupe de francophones 


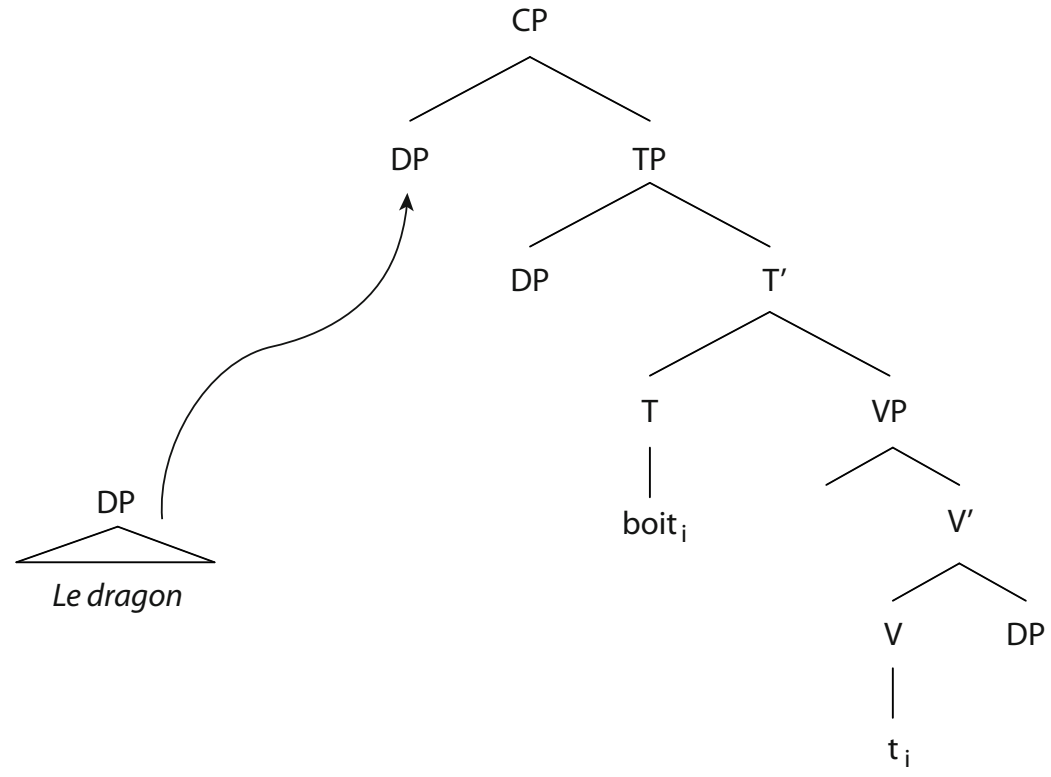

Figure 1. Exemple de substitution

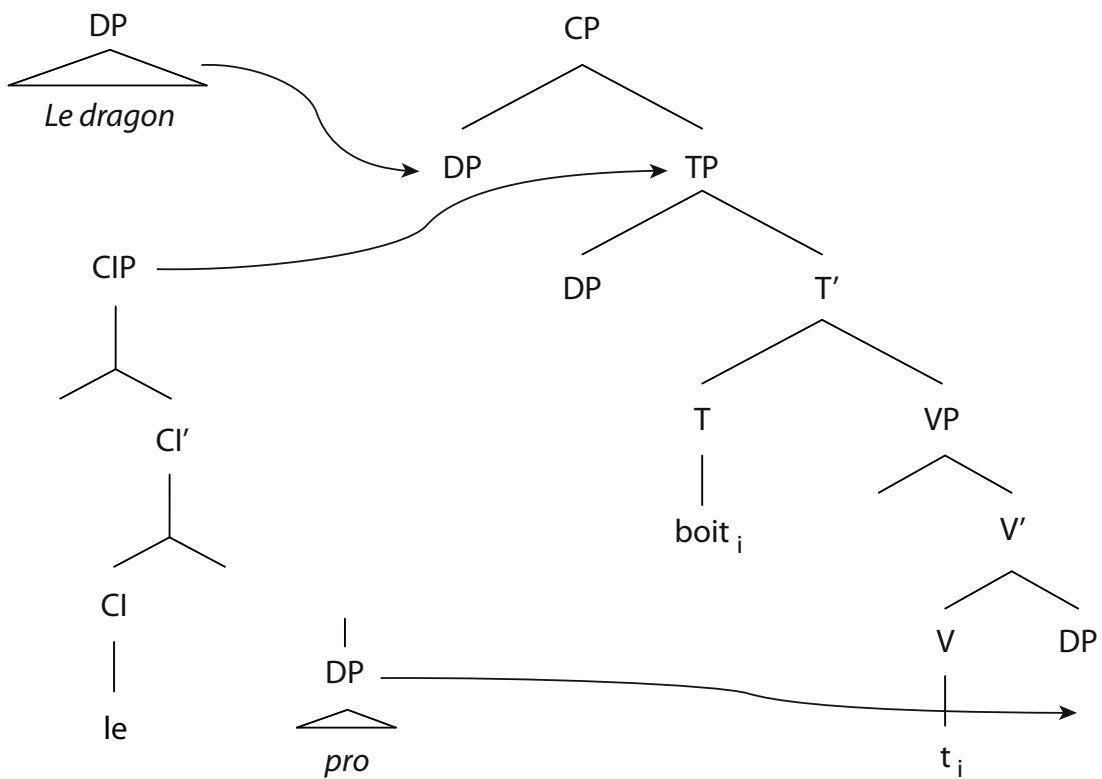

Figure 2. Exemple d'adjonction 
comme groupe de contrôle. Les résultats montrent que le groupe avancé se rapproche du groupe natif en ce sens que ces apprenants arrivent à positionner correctement les clitiques et à acquérir les propriétés de sélection sémantique du verbe, mais ils ne parviennent pas cependant à bien reconnaitre les formes casuelles des clitiques. De fait, les apprenants semblent différencier le datif de l'accusatif, mais ils ont des difficultés à appliquer morphologiquement cette différence. D'autres études ont regardé la maîtrise des clitiques objets directs en L2 (Sneed German et al., 2015; Cuza et al., 2013; Rossi et al., 20I4, entre autres). Sneed German et al. (2015) et Rossi et al. (2014) ont mis en place des expériences de "potentiel évoqué visuel» (PEV - Even Related Potential) combinées avec des jugements d'acceptabilité dont les résultats se rapprochent de la possibilité d'atteindre le niveau des locuteurs natifs (soit en refixant les paramètres en L2: Schwartz et Sprouse, 1994 et 1996; soit par des effets d'usage: Hernandez et al., 2005). Sneed German et al. (2015) ont eu comme population des apprenants en français avec pour Li l'anglais, tandis que Rossi et al. (20I4) ont eu comme population des apprenants en espagnol avec pour Li l'anglais. Les résultats de Sneed German et al. (2015) montrent que les apprenants étaient sensibles aux agrammaticalités syntaxiques et sémantiques des clitiques dans les expériences de PEV au même titre que les natifs même si cela ne se retrouvait pas dans les études offline de tâches métalinguistiques où ils devaient juger de la grammaticalité de phrases, particulièrement pour le groupe avec le niveau en français le plus bas. Rossi et al. (20I4) ont également constaté que les apprenants avancés se montraient sensibles aux agrammaticalités syntaxiques des clitiques et qu'un ensemble d'entre eux était sensible aux agrammaticalités de genre. Cuza et al. (2013) ont mis en place plusieurs expériences de compréhension, de production et de jugement d'acceptabilité avec trois groupes d'apprenants en espagnol ayant pour Li le chinois (des bilingues simultanés, des bilingues précoces et des bilingues tardifs) et un groupe de contrôle de locuteurs natifs de l'espagnol. Les résultats chez les apprenants reflètent finalement un transfert de la Li. Leurs résultats montrent en effet que les bilingues tardifs ont plus de difficultés à produire des clitiques que les autres groupes, et préfèrent recourir aux SNs objets nuls. Ces études s'accordent régulièrement avec «le modèle développemental» (Developmental Model) de Towell et Hawkins (1994). Ces derniers montrent que les apprenants en L2 vont passer par plusieurs étapes et produire différentes formes de clitiques avant d'arriver à la variante correcte. Ils ont repéré à ce propos quatre variantes possibles: l'absence de clitique, le clitique placé en position in situ, le clitique placé après le participe et enfin le clitique placé dans la bonne position syntaxique. L'acquisition en L2 des clitiques montre alors une variété de formes.

Pour autant, les études antérieures diffèrent de notre étude en ce sens que nous proposons une expérience uniquement de production des clitiques objets et réfléchis en français avec trois groupes de population: français, allemand et chinois mandarin. Une autre spécificité par rapport à Grüter et Crago (2012) est que nous avons ajouté un groupe français en tant que groupe de contrôle parce que nous voulions comparer les performances des apprenants en L2 aux performances réelles en Li et non à un standard abstrait du français. 


\subsection{Hypothèses pour la production des clitiques objets chez des apprenants chinois et allemands en français $L 2$}

À partir de ce que nous avons vu sur les différents facteurs intervenant dans l'acquisition des clitiques objets en L2, nous pouvons établir plusieurs hypothèses quant à la production des clitiques avec des prédictions différentes.

Selon l'hypothèse de l'importance de la $\mathrm{L}_{\mathrm{i}}$ en $\mathrm{L}_{2}$, les réponses des apprenants chinois et des apprenants allemands devraient montrer une influence de la Lr. Les apprenants chinois produiraient moins de clitiques et on devrait constater des phrases sans complément d'objet comme cela se retrouve en chinois mandarin, ou alors des phrases avec reprise du complément d'objet. Les apprenants allemands ne devraient également pas systématiquement utiliser des clitiques, et devraient recourir à des types de pronoms comme ceux que nous trouvons en allemand.

Pour l'hypothèse des interfaces, les apprenants chinois comme les apprenants allemands devraient avoir des difficultés à produire des clitiques objets car cette structure semble relever d'interfaces externes et notamment de l'interface syntaxepragmatique, spécialement pour les verbes de type transitif/intransitif pour lesquels l'absence de clitiques n'est pas considérée comme agrammaticale mais inattendue selon le contexte.

L'hypothèse de la «structure de surface» peut prédire deux situations différentes selon la distance prise en compte. En effet, cette hypothèse prédit des difficultés en L2 pour les dépendances non locales. De fait, si nous nous appuyons sur la distance structurale comme celle en figures i et 2, les apprenants devraient avoir des difficultés à produire des clitiques car la dépendance entre le clitique et sa trace est non locale. Cependant, si la distance choisie est linéaire, alors les apprenants ne devraient pas avoir de problèmes à produire des clitiques au même niveau que les natifs puisque la dépendance sera plutôt locale.

Enfin, d'après l'hypothèse sur le rôle de la mémoire de travail, les apprenants en $\mathrm{L}_{2}$ auront des difficultés à produire les clitiques en raison de leur capacité de mémoire de travail. Pour analyser cela, il faut alors mettre en relation leurs productions avec un test sur la capacité de mémoire de travail en Li (pour plus de détails, voir 2.4.I). Certaines erreurs pourront se produire comme l'accord en genre et en nombre du clitique, la position syntaxique incorrecte du clitique ou encore l'omission même du clitique.

\section{Expérience}

\subsection{Objectif}

Nous avons réalisé une expérience permettant d'analyser l'acquisition des clitiques objets français chez des bilingues tardifs dont la Li est le mandarin ou l'allemand et qui ont en moyenne commencé à apprendre leur L2 après l'âge de io ans. Notre objectif est de comprendre comment la construction de ces clitiques est établie 
dans leur interlangue, afin de pouvoir par la suite faire le lien avec les différentes hypothèses en acquisition de L2 (section I.4). L'expérience est un test de production élicitée (voir Eisenbeiss, 20Io) en français. En parallèle, nous avons pris en compte d'autres mesures en proposant un test de mémoire de travail (l'«empan de lecture», Reading Span) en mandarin et en allemand, ainsi qu'un questionnaire à remplir comportant diverses informations concernant la $\mathrm{L}_{2}$ (âge d'acquisition ou fréquence d'utilisation, entre autres).

\subsection{Participants}

Le groupe français est constitué de seize participants, locuteurs natifs du français vivant en France. Ils ont participé à l'expérience en tant que groupe de contrôle. Ils sont âgés de 18 à 30 ans (âge moyen $=23,75$ ).

Le groupe chinois se compose de quinze participants, locuteurs natifs du mandarin. Ils sont âgés de 23 à 26 ans et proviennent de provinces chinoises différentes (âge moyen $=24,4)$. Certains ont un dialecte chinois différent ${ }^{6}$, mais il n'existe dans aucun de ces dialectes des pronoms clitiques objets comme en français. Ces sujets ont des compétences qui les classent entre le niveau Bi et le niveau Ci selon le Cadre européen commun de référence pour les langues (CECRL). Ils sont tous étudiants et habitent en France mais sont nés en Chine. Un participant est classé dans le niveau BI, huit dans le niveau $\mathrm{B}_{2}$, trois dans le niveau $\mathrm{B}_{2} / \mathrm{Cr}_{1}$ et trois dans le niveau $\mathrm{C}_{\mathrm{I}}{ }^{7}$. Les participants ont en moyenne commencé à apprendre le français à partir de 19 ans et 6 mois.

Le groupe allemand comporte douze participants, dont huit résident en Allemagne à Berlin, et quatre en France à Paris, âgés de 20 à 58 ans (âge moyen $=35$ ). La différence d'âge avec le groupe chinois est loin d'être négligeable, car les participants, notamment ceux résidant en Allemagne, ne sont pas des étudiants et prennent des cours de français à l'Institut français de Berlin. Ceci implique alors un apprentissage $\mathrm{du}$ français plus long et influencera sûrement nos résultats pour les performances par rapport au groupe chinois. Les participants allemands ont des compétences les classant entre le niveau $\mathrm{B}_{\mathrm{I}}$ et le niveau $\mathrm{C}_{\mathrm{s}}$ selon le CECRL. Quatre participants relèvent du niveau $\mathrm{B}_{1} / \mathrm{B}_{2}$, trois du niveau $\mathrm{B}_{2} / \mathrm{C}_{1}$, deux du niveau $\mathrm{C}_{\mathrm{I}}$ et trois du niveau $\mathrm{C}_{2}{ }^{8}$. Les participants ont en moyenne commencé à apprendre le français à partir de is ans.

L'écart entre les deux groupes chinois et allemand pour l'âge d'acquisition (comme pour l'âge moyen) est non négligeable, ce qui implique certainement une différence pour la production des clitiques dans l'expérience. Nous reviendrons là-dessus dans la partie «3. Discussion».

6. Entre autres le dialecte du Sichuan, le dialecte de Pékin, le dialecte du Nord-Est, le dialecte Wu, le dialecte de Shaanxi, le dialecte de Shandong, le dialecte de Kumming, le dialecte de Yunnan, le dialecte de Henan, le dialecte de Pinglu, le dialecte de Shanxi et le dialecte de Chongqing.

7. Cette classification est faite selon leurs diplômes antérieurs. Nous ne leur avons pas fait passer de test de français.

8. À nouveau, cette classification est faite selon leurs diplômes antérieurs. Nous ne leur avons pas fait passer de test de français. 


\subsection{Expérience: test de production élicitée}

\subsubsection{Caractéristiques}

Cette expérience s'inspire de celle de Grüter et Crago (20I2) pour le test de production. Nous utilisons des histoires racontées avec comme support des images. La tâche est de répondre à une question. Cela étant, l'expérience de Grüter et Crago ne comportait que des verbes avec des compléments d'objet pour inciter la production d'un clitique objet ( $Q$ Que fait Sophie avec le chien? - Elle le brosse»). Nous avons voulu aller plus loin en examinant la production de clitiques selon le contexte favorisé (contextes transitif, intransitif, réfléchi) et le type de verbes (verbes transitifs/intransitifs et transitifs/réfléchis). En français, des verbes peuvent impliquer la production de clitiques objets ou non (transitif/intransitif comme manger) ou la production de clitiques objets ou réfléchis (transitif/réfléchi comme brosser). Ainsi, nous avons voulu analyser la production des clitiques objets en tenant compte du type de verbes, ce qui nous a conduit à regarder la production des clitiques objets de forme réfléchie, même s'il est indéniable que ce clitique a certaines caractéristiques différentes du clitique objet comme le, la et les. Contrairement à l'expérience de Grüter et Crago (20I2), nous avons également fait passer l'expérience à un groupe de locuteurs du français Li comme groupe de contrôle afin de mieux comparer les résultats en $\mathrm{L}_{2}$ avec une performance native qui ne suit pas forcément le français standard. Comme notre expérience concerne des adultes, nous avons pris un autre modèle de test de mémoire (une version de l'«empan de lecture», que nous allons détailler dans 2.4.I) que ceux mis en place chez Grüter et Crago, plus adaptés pour les enfants.

\subsubsection{Déroulement}

Chaque participant devait écouter 33 histoires de is secondes environ, chacune affichée sur un PowerPoint ${ }^{9}$. Toutes les histoires étaient composées de trois images, à savoir deux animaux, et un lieu ou un objet. Les images étaient positionnées de la même manière pour toutes les histoires, avec les animaux à gauche et à droite, et le lieu ou l'objet en haut au centre. Le texte de l'histoire apparaissait durant l'écoute. À la fin, une question s'affichait portant sur l'action d'un des deux animaux (figure 3). Le participant devait y répondre en une seule phrase de la manière la plus courte possible, en utilisant le clitique sujet en début de phrase ( $i l$ ou elle) et en prenant le verbe indiqué entre parenthèses apparaissant à côté de la question. Ces questions incitaient alors la réponse appropriée selon le contexte linguistique (favorisation ou non de clitiques). Par exemple, la question "Qu'est-ce que fait le dragon avec l'eau de la rivière?» implique la réponse la plus naturelle et la plus courte possible «Il la boit», favorisant la production du clitique objet dans ce contexte. Le test de production était donné au tout début de la session.

9. Le matériel est donné en annexe I. 

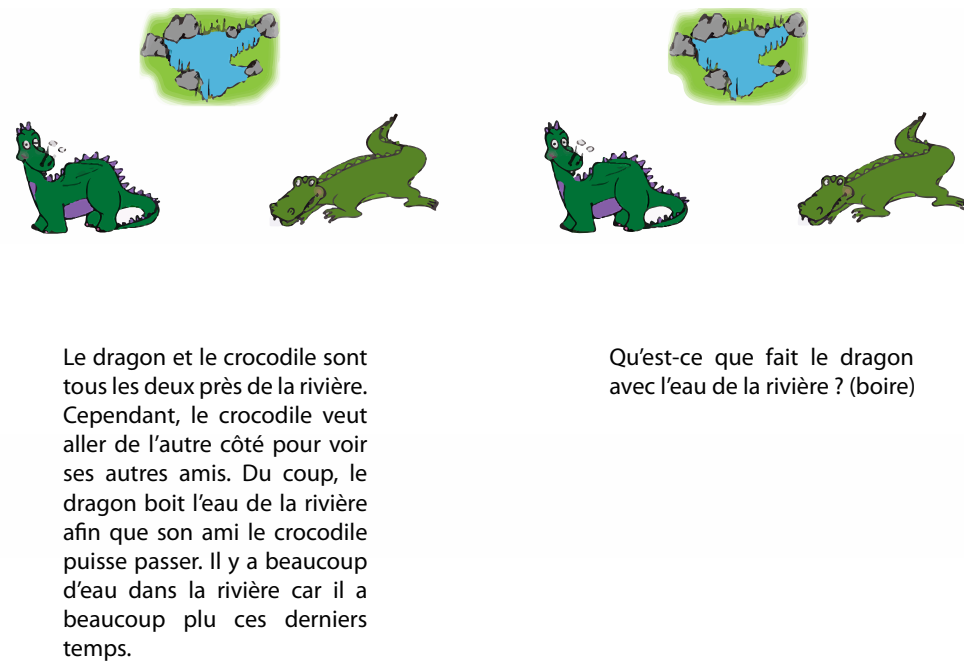

Qu'est-ce que fait le dragon avec l'eau de la rivière? (boire)

Figure 3. Exemple d'item du test de production élicitée

\subsubsection{Matériel}

Quatre conditions ont été testées avec deux types de verbes: le premier type de verbes représente ceux qui peuvent être transitifs ou intransitifs selon le contexte (comme manger ou boire). Le second type de verbes concerne ceux étant transitifs ou réfléchis selon le contexte (comme brosser ou blesser). Huit verbes représentent le premier type à conditions ( $\mathrm{I}$ ) transitif et (2) intransitif, et huit verbes constituent le second type à conditions (3) transitif et (4) réfléchi. Dix-sept distracteurs ont été ajoutés qui sont des verbes principalement intransitifs. Deux listes ont ainsi été élaborées à partir du modèle du carré latin (encadrés I et 2). Les items étaient quasi randomisés pour éviter d'avoir deux items expérimentaux à la suite. La même randomisation a été utilisée pour tous les participants.

\footnotetext{
Condition (I) transitif

Le dragon et le crocodile sont tous les deux près de la rivière. Cependant, le crocodile veut aller de l'autre côté pour voir ses autres amis. Du coup, le dragon boit l'eau de la rivière afin que son ami le crocodile puisse passer. Il y a beaucoup d'eau dans la rivière car il a beaucoup plu ces derniers temps. Qu'est-ce que fait le dragon avec l'eau de la rivière? (boire)

Condition (2) intransitif

Le dragon et le crocodile sont tous les deux près de la rivière. Cependant, le crocodile veut aller de l'autre côté pour voir ses autres amis. Mais le dragon ne veut pas bouger car il a très soif. Du coup, il boit et se sent bien mieux. Le crocodile rejoint pendant ce temps ses amis de l'autre côté de la rivière. Il y a beaucoup d'eau dans la rivière car il a beaucoup plu ces derniers temps. Qu'est-ce que fait le dragon? (boire)
}

Encadré 1. Exemple de verbes à conditions (1) transitif et (2) intransitif 


\section{Condition (3) transitif}

Le singe et le chat sont voisins. Le singe veut faire une farce au chat. Ainsi, il réussit à faire entrer le chat dans le carton puis baisse les côtés du carton pour enfermer le chat. Le chat ne comprend pas ce qu'il se passe et a très peur.

Qu'est-ce que fait le singe avec les côtés du carton? (baisser)

Condition (4) réfléchi

Le singe et le chat sont voisins. Le singe veut faire une farce au chat en se cachant dans le carton. Le chat ne retrouve plus le singe et s'inquiète. Du coup, le chat baisse sa tête pour voir ce qu'il y a dans le carton et aperçoit le singe.

Qu'est-ce que fait le chat pour voir ce qu'il y a dans le carton? (baisser)

Encadré 2. Exemple de verbes à conditions (3) transitif et (4) réfléchi

\subsection{Autres mesures prises en compte en parallèle de l'expérience}

\subsubsection{Test de mémoire de travail}

L'«empan de lecture» choisi est basé sur le modèle de Daneman et Carpenter (1980). Ce modèle d'«empan de lecture» semble être le plus adapté pour notre expérience puisqu'il concerne le traitement des phrases et, selon Daneman et Hannon (2007), il montre d'ordinaire une bonne corrélation à la fois avec la compréhension des textes et avec d'autres tests de mémoire de travail (tel que l'«empan d'opération», Operation Span).

Ce test de mémoire a été présenté sous la forme d'un PowerPoint, les consignes et les exemples étaient en mandarin ou en allemand selon les participants. Le test n'a alors en aucun cas pour objectif de refléter la compétence en L2 mais cherche à mesurer la mémoire de travail. Il était toujours proposé en fin de session afin d'éviter toute interférence de la Li pour le test de production élicitée et de rester le plus possible dans un contexte linguistique français. Des explications en français à l'oral étaient ajoutées si nécessaire. Le test consistait en 60 phrases. Les sujets devaient répéter le dernier mot de chaque phrase, qui était un mot lexical. Le sujet devait lire à voix haute d'abord deux phrases de suite, puis répéter les derniers mots, ensuite trois phrases, quatre phrases, cinq phrases et enfin six phrases. Il y avait à chaque fois trois groupes de deux, trois, quatre, cinq, six phrases. Le participant ne devait commencer en aucun cas par le dernier mot qu'il avait entendu. Si tel était le cas, le mot n'était pas comptabilisé. Le score obtenu pour l'«empan de lecture» correspondait au nombre de mots correctement répétés.

\subsubsection{Questionnaire d'histoire linguistique}

Les participants chinois et allemands ont rempli un questionnaire concernant leur profil linguistique ( $\mathrm{Li}$ et al., 2013). Les participants chinois l'ont rempli en ligne à la maison. Les participants allemands ont rempli ce questionnaire sur place. Cela permettait, entre autres, de savoir leur âge, leur arrivée en France, leur niveau en français, leur âge d'acquisition, les autres langues parlées, le temps consacré au français comparé à la Li (chinois ou allemand). Ce questionnaire est extrait du site «Brain, 
Language, and Computation Lab» (http://blclab.org/language-history-questionnaire/) et est une forme réduite du questionnaire en ligne de Li et al. (2013).

\subsection{Résultats}

\subsubsection{Hypothèses selon les théories évoquées}

Pour le groupe français, en tant que groupe de contrôle, nous devrions avoir une production attendue de clitiques dans toutes les conditions: clitique objet dans les conditions (I) et (3), réfléchi dans la condition (4) et pas de clitique dans la condition (2). Pour le français L2, les hypothèses énoncées en I.4 vont prédire des résultats différents pour la production de clitiques dans les quatre conditions.

Selon l'hypothèse (a) de la «structure de surface», les groupes chinois et allemand en L2 devraient montrer une production similaire à celle du groupe de contrôle si la distance prise en compte est linéaire et donc la dépendance locale. Si la distance est cependant structurale, la production des groupes chinois et allemand devrait être différente de celle du groupe français puisque les dépendances seront non locales, rendant la production de clitiques plus difficile.

$\mathrm{Si}$, selon l'hypothèse (b), l'influence de la Li est déterminante, nous devrions constater une omission relativement fréquente de l'objet (clitique ou SN) chez les sujets chinois ainsi qu'une reprise du SN chez les sujets allemands, voire l'utilisation du clitique comme un pronom allemand, soit dans la position initiale du SN objet.

L'hypothèse (c) sur les interfaces devrait créer une différence entre le groupe de contrôle et les deux autres groupes, ces deux derniers produisant peu de phrases avec une utilisation attendue des clitiques si l'on considère que les clitiques relèvent en partie de l'interface pragmatique, notamment les clitiques objets directs le, la et les. Les résultats pourront alors prendre les formes suivantes: une non-production de clitiques, la reprise du SN objet, l'utilisation du clitique en position postverbale, ou encore des problèmes d'accord en genre et en nombre.

Enfin, les groupes chinois et allemands devraient rencontrer plus de difficultés que le groupe de contrôle selon l'hypothèse (d) à propos du rôle de la mémoire de travail, et des erreurs devraient avoir lieu comme celles que l'on retrouve dans les autres hypothèses. À l'appui de cela, une corrélation devrait se dessiner entre la production des clitiques et les résultats de l'«empan de lecture» qui mesure la mémoire de travail (voir 2.4.I pour plus d'explications sur sa fonction).

\subsubsection{Analyse des erreurs pour les trois groupes}

Tous les résultats ont été analysés avec le logiciel $\mathrm{R}$ au moyen de modèles linéaires mixtes généralisés (Baayen et al., 2008), plus spécifiquement les modèles de régression logistique, en utilisant la fonction «glmer» (package «lme4»). Pour nos analyses, les variables indépendantes adoptées sont les quatre conditions manipulées - (I) transitif, (2) intransitif pour les verbes transitifs/intransitifs, (3) transitif, (4) réfléchi pour les verbes transitifs/réfléchis - ainsi que les trois groupes de locuteurs - chinois, allemand 
et français -, les items et les sujets étant les variables aléatoires. La variable dépendante est la production ou non de clitiques. Afin de faciliter la comparaison des conditions, nous avons fait des sous-ensembles par type de verbes (verbes transitifs/intransitifs et verbes transitifs/réfléchis).

Nous avons au préalable analysé les diverses réponses possibles par les participants, c'est-à-dire que nous avons tenu compte du «modèle développemental» de Towell et Hawkins (1994) en prenant en considération les différentes variantes possibles de la réalisation du clitique, soit, dans ce cadre, les clitiques utilisés dans les conditions non favorables, les clitiques conjugués de manière incorrecte et/ou placés dans une position syntaxique erronée, c'est-à-dire après le verbe en position in situ. Cela étant, nous avons constaté que les clitiques postverbaux n'étaient pas utilisés chez les sujets allemands, comme chez les sujets chinois. De fait, la production inattendue contenait majoritairement la non-production de clitiques. Comme le montre le tableau I, une petite partie concernait la production de clitiques objets dans une condition inattendue ( $8 / \mathrm{I} 88$ pour le groupe allemand, $2 / 238$ pour le groupe chinois et $4 / 256$ pour le groupe français) ou la production de clitiques avec un accord incorrect en genre ( $\mathrm{IO} / \mathrm{I} 88$ pour le groupe allemand, $5 / 238$ pour le groupe chinois et $0 / 256$ pour le groupe français); c'est pour cela que nous avons décidé de ne pas les analyser séparément et de les regrouper soit dans la production attendue de clitiques soit dans la production non attendue de clitiques. Nous avons cependant considéré comme attendue la production de clitiques avec un accord incorrect en genre, puisque cela ne reflète pas directement la compréhension et la maittrise de cette catégorie syntaxique pour les apprenants mais plutôt un problème d'acquisition lexicale. Nous avons analysé également la reprise du SN objet au lieu du clitique. Par rapport à cela, nous avons comptabilisé le SN objet seulement si celui-ci était le même que celui mentionné dans le texte ou un synonyme. De fait, nous avons adopté une approche binaire (production attendue $v$ s production inattendue) pour les clitiques et pour les SNs objets.

\begin{tabular}{|c|c|c|c|c|c|c|}
\hline Groupes & $\begin{array}{l}\text { Productions } \\
\text { attendues } \\
\text { de clitiques }\end{array}$ & $\begin{array}{l}\text { SNs } \\
\text { produits } \\
\text { au lieu } \\
\text { des } \\
\text { clitiques }\end{array}$ & $\begin{array}{l}\text { SNs non } \\
\text { attendus } \\
\text { ou } \\
\text { incorrects }\end{array}$ & $\begin{array}{l}\text { Clitiques non } \\
\text { attendus dans } \\
\text { les conditions } \\
\text { (réfléchi ou } \\
\text { indirect au } \\
\text { lieu du clitique } \\
\text { objet...) }\end{array}$ & $\begin{array}{l}\text { Clitiques } \\
\text { mal } \\
\text { accordés }\end{array}$ & $\begin{array}{l}\text { Non- } \\
\text { production } \\
\text { de } \\
\text { clitiques } \\
\text { ou de SNs }\end{array}$ \\
\hline Allemand & $\begin{array}{r}52,7 \% \\
(99 / 188)\end{array}$ & $\begin{array}{r}7,5 \% \\
(\mathrm{I} 4 / \mathrm{I} 88)\end{array}$ & $\begin{array}{r}\mathbf{1}, 6 \% \\
(3 / 188)\end{array}$ & $\begin{array}{r}4,3 \% \\
(8 / 188)\end{array}$ & $\begin{array}{r}5,3 \% \\
(\mathrm{IO} / \mathrm{I} 88)\end{array}$ & $\begin{array}{r}\mathbf{2 8 , 7} \% \\
(54 / \mathrm{i} 88)\end{array}$ \\
\hline Chinois & $\begin{array}{r}37,4 \% \\
(89 / 238) \\
\end{array}$ & $\begin{array}{r}\text { IO,I \% } \\
(24 / 238) \\
\end{array}$ & $\begin{array}{r}2,5 \% \\
(6 / 238) \\
\end{array}$ & $\begin{array}{r}0,8 \% \\
(2 / 238) \\
\end{array}$ & $\begin{array}{r}\mathbf{2 , \mathbf { I }} \% \\
(5 / 238) \\
\end{array}$ & $\begin{array}{r}47, \mathbf{I} \% \\
(\mathrm{II} 2 / 238) \\
\end{array}$ \\
\hline Français & $\begin{array}{r}82,8 \% \\
(212 / 256)\end{array}$ & $\begin{array}{r}2 \% \\
(5 / 256)\end{array}$ & $\begin{array}{r}0,4 \% \\
(\mathrm{I} / 256)\end{array}$ & $\begin{array}{r}\text { I,6 \% } \\
(4 / 256)\end{array}$ & $\begin{array}{r}0 \% \\
(0 / 256)\end{array}$ & $\begin{array}{r}13,3 \% \\
(34 / 256)\end{array}$ \\
\hline
\end{tabular}

Tableau 1. Erreurs commises par les participants pour chaque groupe 


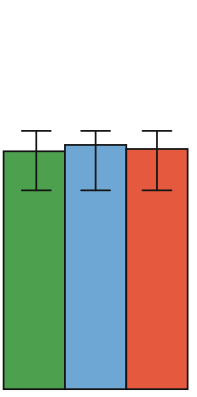

(2) intransitif

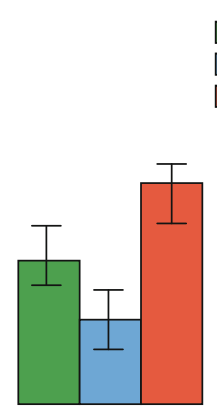

(3) transitif
Allemand

Chinois

Français

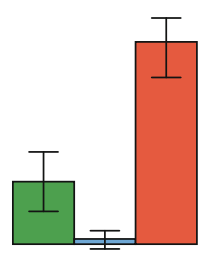

(4) réfléchi

Verbes transitifs/intransitifs $(1,2)$ et transitifs/réfléchis $(3,4)$

Figure 4. Fréquence de production attendue de clitiques pour les groupes chinois, allemand et français; les barres d'erreurs reflètent des intervalles de confiance (95\%)

Nous avons alors considéré que la production de clitiques est attendue si elle correspond aux attentes du contexte. Nous avons noté i pour la production d'un clitique attendu selon le contexte et o dans le cas contraire. De fait, pour la condition (I) et la condition (3) transitif, le clitique attendu est un clitique objet le, la ou les. La production attendue de clitiques dans la condition (2) est aucune, ce qui signifie qu'aucun clitique dans cette condition ne correspond à la production attendue, donc cela sera noté I. Enfin, la condition (4) implique comme production attendue la réalisation du clitique réfléchi se pour être notée I.

\subsubsection{Production attendue de clitiques pour les trois groupes}

Nous avons observé la production attendue de clitiques pour les trois groupes testés. Les locuteurs natifs du français réussissent en moyenne $83 \%$ pour la production de clitiques attendus. La production attendue de clitiques selon les conditions pour les niveaux $\mathrm{B}_{1}, \mathrm{~B}_{2}, \mathrm{~B}_{2} / \mathrm{C}_{\mathrm{I}}$ et $\mathrm{C}_{\mathrm{I}}$ dans le groupe chinois est respectivement de $25 \%, 32 \%$, $49 \%$ et $54 \%$, alors que la production attendue pour les niveaux $\mathrm{BI}_{\mathrm{I}} / \mathrm{B}_{2}, \mathrm{~B}_{2} / \mathrm{CI}_{\mathrm{I}}$, $\mathrm{CI}$ et $\mathrm{C}_{2}$ dans le groupe allemand est respectivement de $42 \%, 56 \%, 47 \%$ et $88 \%{ }^{10}$.

La figure 4 montre les résultats pour les quatre conditions parmi les trois groupes (allemand, français et chinois). Une différence significative est constatée entre les groupes. En effet, on observe que les sujets allemands produisent moins de clitiques

10. Comme nous n'avons pas testé leur niveau de français, il faut noter que nous ne pouvons pas être sûrs que les participants connaissaient tous les verbes, et notamment les formes réflexives, comme l'a fait remarquer un relecteur. Les verbes étaient en revanche tous assez fréquents selon la base de données «Lexique 3» (lexique.org) - fréquence moyenne d'occurrences $=490$ sur 15 millions de mots - et devraient généralement faire partie du vocabulaire $\mathrm{B}_{2}+$. 
attendus que les sujets français $(\beta=-\mathrm{I}, 5585, z=-3,66 \mathrm{I}, p<0$, O०I $)$, et il en est de même pour les sujets chinois $(\beta=-2,5230, z=-6,191, p<0,00 \mathrm{I})$. Les participants français réussissent à mieux produire les clitiques dans les contextes attendus que les participants chinois et allemands, comme cela était supposé. Également, on trouve une différence significative entre le groupe chinois et le groupe allemand $(\beta=0,9646, z=2,337, p<0,05)$, les sujets allemands arrivant plus facilement à produire des clitiques attendus que les sujets chinois.

\subsubsection{Production attendue chez le groupe français}

Nous avons analysé la production de clitiques attendue dans les quatre conditions pour le groupe français. La figure 5 présente les résultats des sujets français pour toutes les conditions selon le type de verbes (transitif/intransitif vs transitif/réfléchi).

Le type de verbes n'influence en rien la production attendue de clitiques pour les sujets français puisqu'il n'y a pas de différence significative dans la production attendue de clitiques entre les verbes transitifs/intransitifs et les verbes transitifs/ réfléchis ( $\beta=0,07012, z=0,248$, ns; moyenne de production attendue pour transitif/ intransitif $=0,8 \mathrm{I}$; moyenne de production attendue pour transitif/réfléchi $=0,85$ ). Cependant, une différence significative est constatée quand on regarde séparément les deux classes de verbes (transitif/intransitif et transitif/réfléchi) entre la condition (I) (transitif pour les verbes transitif/intransitifs) et la condition (2) (intransitif pour les verbes transitifs/intransitifs) $(\beta=1,7170, z=3,905, p<0,00 \mathrm{I})$, ce qui n'est pas le cas entre la condition (3) (transitif pour les verbes transitifs/réfléchis) et la condition (4) (réfléchi pour les verbes transitifs/réfléchis), la différence étant seulement numérique ( $\beta=-0,4300, z=-\mathrm{I}, 423, \mathrm{~ns})$.

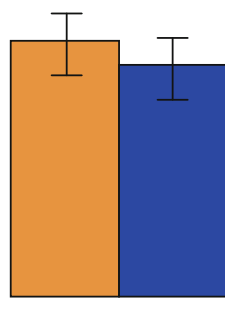

transitif/réfléchi

Verbes transitifs/intransitifs $(1,2)$ et transitifs/réfléchis $(3,4)$

Figure 5. Fréquence de production attendue de clitiques pour le groupe français; les barres d'erreurs reflètent les intervalles de confiance de $95 \%$ 
En comparant toutes les conditions entre elles, on constate que la condition (I) (transitif pour les verbes transitifs/intransitifs) est la moins bien réussie pour la production attendue de clitiques par rapport aux conditions (2) (intransitif pour les verbes transitifs/intransitifs) $(\beta=3,3385, z=4,017, p<0,00 \mathrm{I})$, (3) (transitif pour les verbes transitifs/réfléchis) $(\beta=1,9274, z=3,049, p<0$, OI), et (4) (réfléchi pour les verbes transitifs/réfléchis) $(\beta=1,1306, z=2,055, p<0,05)$, ce qui peut sembler dans un premier temps surprenant puisque le contexte incite à la production de clitiques (nous reviendrons là-dessus dans la partie «3. Discussion»). Une différence significative est aussi notée entre la condition (2) et les conditions (4) $(\beta=-2,2079$, $z=-2,517, p<0,05)$ et (I) $(\beta=-3,3384, z=-4,017, p<0,00 \mathrm{I})$. La condition (2) qui demande la non-production d'un pronom clitique semble ainsi être la plus facile même pour les locuteurs natifs.

\subsubsection{Production attendue chez le groupe chinois}

Nous avons ensuite comparé la production des clitiques entre les deux groupes français et chinois. La figure 6 montre les résultats des sujets français et des sujets chinois pour les quatre conditions selon le type de verbes (transitif/intransitif vs transitif/réflécbi).

Une différence significative est observée entre les deux groupes de locuteurs, c'està-dire que les sujets français ont une meilleure production attendue de clitiques que les sujets chinois pour les deux types de verbes, soit transitif/intransitif $(\beta=1,0104$, $z=3, \mathrm{I} 45, p<0$, OI $)$ et transitif/réfléchi $(\beta=4,6767, z=5,634, p<0,0 \mathrm{OI})$.

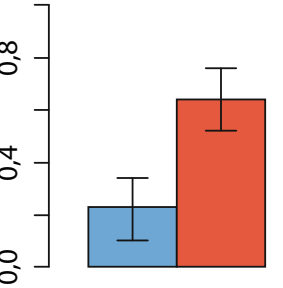

(1) transitif

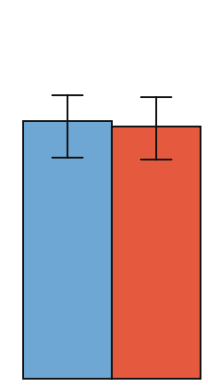

(2) intransitif

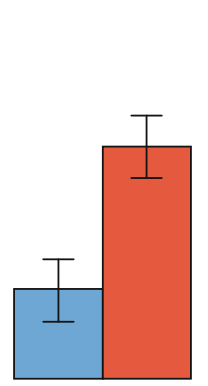

(3) transitif
Chinois

Français

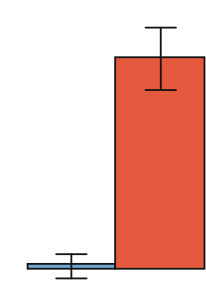

(4) réfléchi

Verbes transitifs/intransitifs $(1,2)$ et transitifs/réfléchis $(3,4)$

Figure 6. Fréquence de production attendue de clitiques objets selon les conditions pour les groupes chinois et français; les barres d'erreurs reflètent des intervalles de confiance $(95 \%)$ 


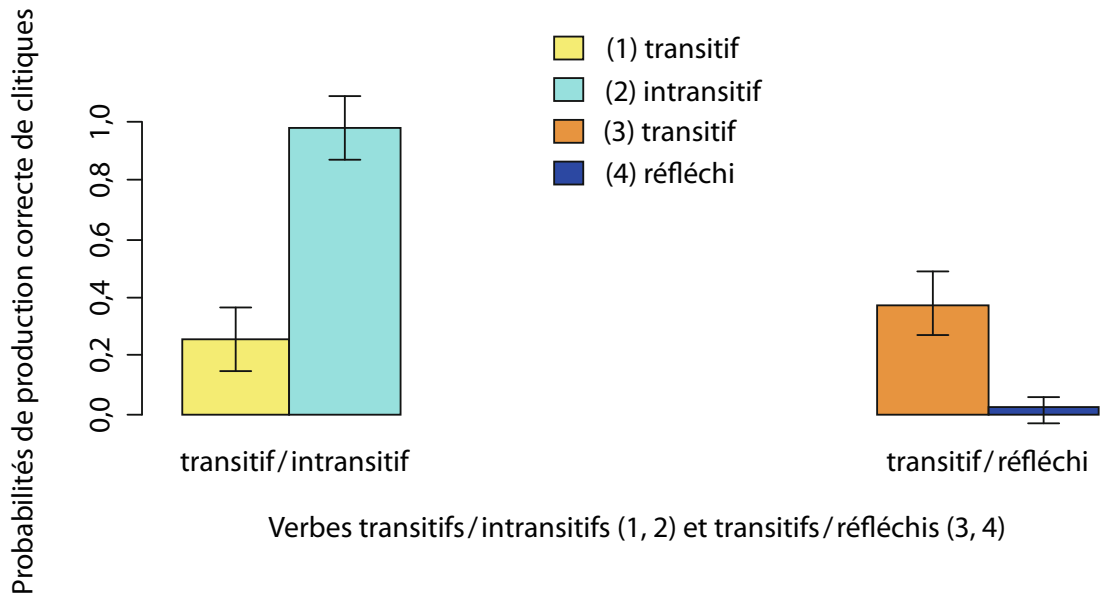

Figure 7. Fréquence de production attendue de clitiques pour le groupe chinois; les barres d'erreurs reflètent des intervalles de confiance $(95 \%)$

Nous avons ensuite analysé la production des clitiques dans les quatre conditions uniquement pour le groupe chinois. Pour les sujets chinois, la figure 7 offre des patterns similaires pour la production de clitiques à ceux des sujets français, mis à part pour la condition (4) et mis à part une production de clitiques généralement plus faible. Une différence significative est cependant observée entre les deux types de verbes $(\beta=2,9548, z=4,725, p<0$, ooI; moyenne de production attendue pour transitif/intransitif $=0,6 \mathrm{I}$; moyenne de production attendue pour transitif/réfléchi $=0,18)$, les verbes de type transitif/réfléchi étant les plus difficiles à traiter. Pour les verbes de type transitif/intransitif, la différence entre les deux conditions (I) et ( 2 ) est significative $(\beta=3,4529, z=4,404, p<0$, O०I), la condition (I) étant la plus difficile à réaliser. En ce qui concerne les verbes transitifs/réfléchis, les sujets chinois produisent moins de clitiques attendus dans la condition (4) réfléchi $(\beta=-5,667, z=-2,392, p<0,05)$. On observe à ce propos une meilleure production attendue pour la condition (I) $(\beta=4,179, z=2,912$, $p<\mathrm{O}, \mathrm{OI})$, la condition ( 2$)(\beta=\mathrm{I} 2,946, z=5,343, p<0, \mathrm{OOI})$ et la condition (3) $(\beta=5,305, z=3,702, p<0,00 \mathrm{I})$, par rapport à la condition $(4)$, la production du clitique réfléchi étant la plus difficile à maîtriser.

Par ailleurs, nous avons regardé pour le groupe chinois la production de SNs objets à la place de la production attendue de clitiques. La figure 8 montre les résultats des sujets chinois et des sujets français pour les SNs objets dans chaque condition selon le type de verbes. On constate que les sujets chinois vont produire des SNs objets et non nécessairement des phrases agrammaticales. Cette stratégie du SN pour éviter la production de clitiques est dans l'ensemble numériquement moins importante en français $\operatorname{Li}(\beta=-\mathrm{I}, 454, z=-\mathrm{I}, 192, \mathrm{~ns})$. 


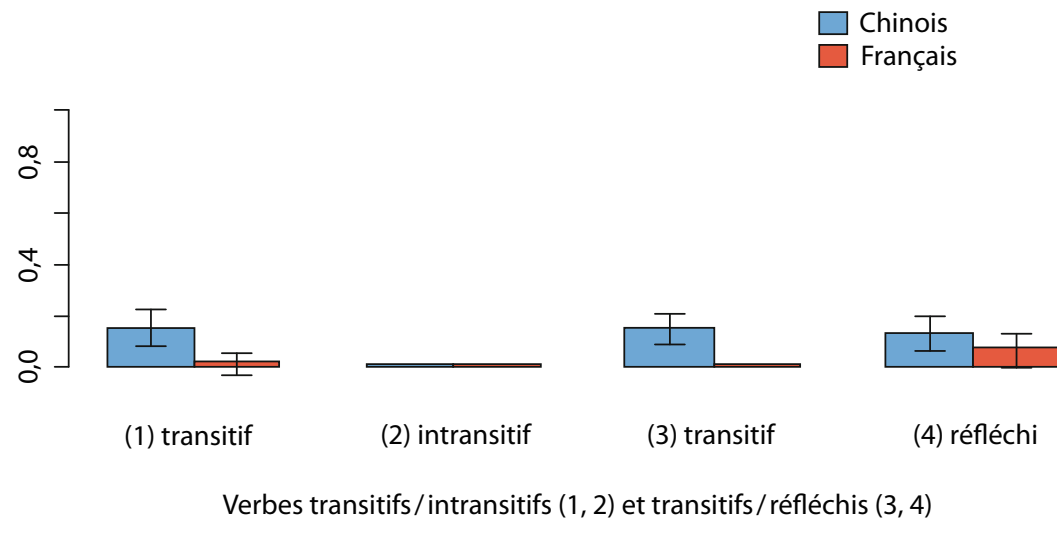

Figure 8. Fréquence de production attendue de SNs objets selon les conditions pour les deux groupes; les barres d'erreurs reflètent des intervalles de confiance (95\%)

\subsubsection{Production attendue chez le groupe allemand}

Nous avons comparé la production de clitiques entre le groupe allemand et le groupe français pour toutes les conditions selon le type de verbes. Selon la figure 9, la différence entre le groupe allemand et le groupe français se remarque particulièrement pour les verbes de type transitif/réfléchi où la différence est significative $(\beta=2,7520$, $z=4,318, p<0,00 \mathrm{I})$. Le groupe allemand produit effectivement moins de clitiques attendus pour ce type de verbes que le groupe français, contrairement aux verbes de type transitif/intransitif pour lesquels aucune différence significative n'est constatée $(\beta=0,3590, z=0,718, \mathrm{~ns})$.

Nous avons par la suite analysé la production de clitiques dans toutes les conditions selon le type de verbes seulement dans le groupe allemand. Comme en chinois mandarin, on observe avec la figure io que le type de verbes joue un rôle significatif ( $\beta=\mathrm{I}, 4 \mathrm{I} 7 \mathrm{O}, z=4,596, p<0,00 \mathrm{I})$, à savoir que les conditions (I) et (2) des verbes de type transitif/intransitif regroupent plus de productions attendues que les conditions (3) et (4) des verbes de type transitif/réfléchi (moyenne de production attendue pour transitif/intransitif $=0,75$, moyenne de production attendue pour transitif/réfléchi $=0,4 \mathrm{I})$. Pour les verbes transitifs/intransitifs, la différence entre les deux conditions (I) et (2) est significative $(\beta=2,2078, z=3$,I2I, $p<0,0 \mathrm{I})$, la condition (I) étant plus difficilement réalisée comme attendue que la condition (2). La production des clitiques pour les verbes transitifs/réfléchis montre également des différences significatives, les sujets allemands produisant moins souvent les clitiques attendus dans la condition (4) $(\beta=-\mathrm{I}, \mathrm{I} 254, z=-3,307, p<0, \mathrm{OOI})$ que dans la condition (3), comme les sujets chinois. La production de clitiques attendus dans la condition (4) réfléchi est à ce propos significativement plus basse que dans les conditions (I) $(\beta=\mathrm{I}, 9105, z=3,05 \mathrm{I}$, $p<0, \mathrm{OI}),(2)(\beta=5,8904, z=5,408, p<0,00 \mathrm{I})$ et $(3)(\beta=2, \mathrm{I} 329, z=3,498, p<0, \mathrm{OOI})$. 


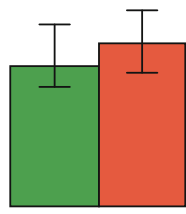

(1) transitif

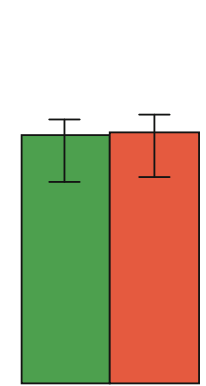

(2) intransitif

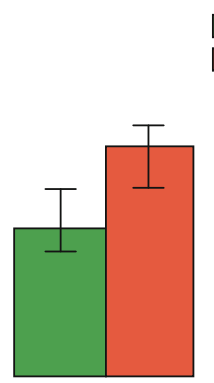

(3) transitif
Allemand

Français

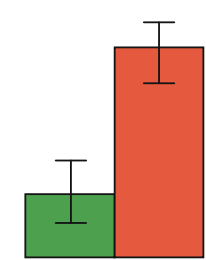

(4) réfléchi

Verbes transitifs/intransitifs $(1,2)$ et transitifs/réfléchis $(3,4)$

Figure 9. Fréquence de production attendue de clitiques objets selon les conditions pour les deux groupes, français et allemand; les barres d'erreurs reflètent des intervalles de confiance $(95 \%)$

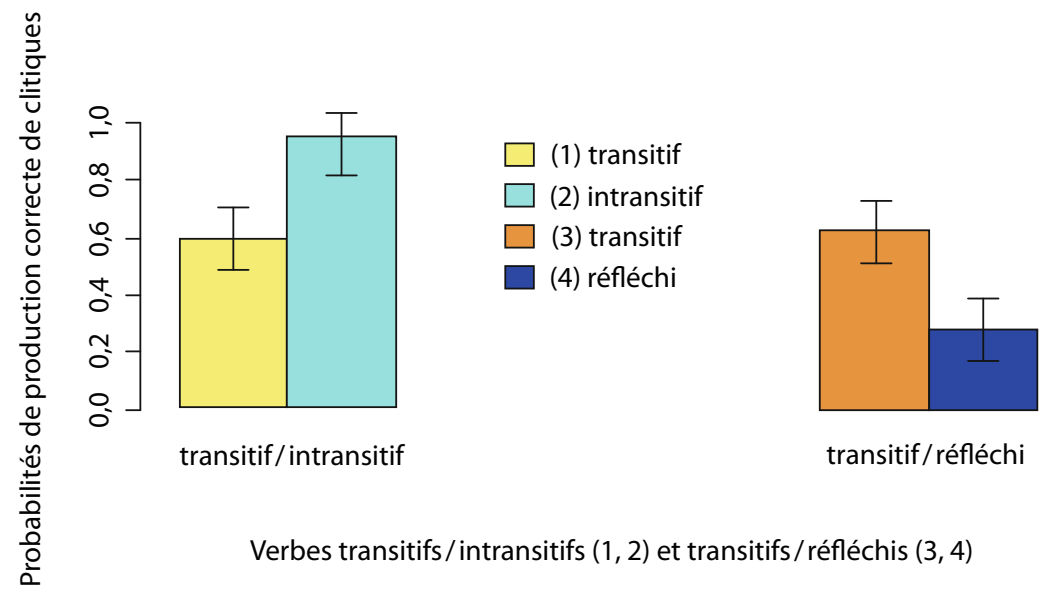

Figure 10. Fréquence de production attendue de clitiques pour le groupe allemand; les barres d'erreurs reflètent des intervalles de confiance (95\%)

La figure in rejoint la figure 8 concernant les sujets chinois en ce sens que les sujets allemands peuvent également avoir comme stratégie de produire des SNs objets à la place des clitiques et non forcément des phrases agrammaticales. Cela est d'ailleurs dans l'ensemble numériquement moins important en français LI $(\beta=-\mathrm{I}, 467, z=-\mathrm{I}, 34 \mathrm{I}, \mathrm{ns})$. 


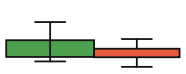

(1) transitif
(2) intransitif

Allemand

Français

Verbes transitifs/intransitifs $(1,2)$ et transitifs/réfléchis $(3,4)$

Figure 11. Fréquence de production attendue de SNs objets selon les conditions pour les deux groupes; les barres d'erreurs reflètent des intervalles de confiance (95\%)

\subsubsection{Comparaison entre les groupes allemand et chinois}

Il est intéressant de constater que les tendances de production des clitiques en français L2 sont similaires pour les deux groupes chinois et allemand, ce que montre la figure I2. En effet, les deux groupes ne produisent presque pas le clitique dans la condition (2), ce qui est attendu, et ils éprouvent des difficultés avec la production du clitique réfléchi dans la condition (4), surtout le groupe chinois. Les résultats pour le groupe chinois sont moins proches numériquement et significativement de ceux du groupe allemand dans la condition (I) $(\beta=-2,9833, z=-\mathrm{I}, 9 \mathrm{I} 4, p<0, \mathrm{I})$ et dans la condition (4) $(\beta=-5,632, z=-2,035, p<0,05)$ mais pas dans les autres conditions. Il faut par ailleurs tenir compte du fait que le niveau en français est légèrement plus élevé dans le groupe allemand que dans le groupe chinois. Les plus faibles dans le groupe allemand ont le niveau $\mathrm{B}_{1} / \mathrm{B}_{2}$ et les plus forts le niveau $\mathrm{C}_{2}$ alors que le niveau le plus faible et le plus fort sont respectivement $\mathrm{BI}_{1}$ et $\mathrm{C}_{2}$ pour les sujets chinois.

\subsubsection{Corrélations pour les sujets chinois}

Afin de déterminer si certains facteurs ont une influence sur la production de réponses attendues chez les sujets chinois, nous avons effectué des analyses concernant la corrélation entre les réponses de clitiques objets corrects et les autres facteurs potentiels comme l'«empan de lecture», le niveau en français ( $\mathrm{B}_{1}, \mathrm{~B}_{2}, \mathrm{~B}_{2} / \mathrm{C}_{1}, \mathrm{C}_{\mathrm{I}}$ ), l'année du début d'apprentissage ou la date d'arrivée en France ${ }^{11}$. Une corrélation positive entre la mémoire de travail et la production attendue de clitiques est constatée $(r=0,22, p<0,0 \mathrm{I})$

11. En raison d'un problème technique, un participant chinois a été exclu pour ces analyses, car nous n'avions pas ses données. 


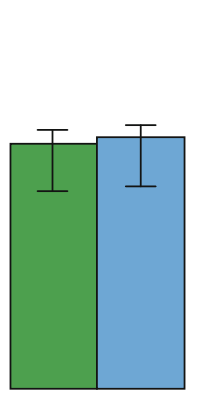

(1) transitif

(2) intransitif

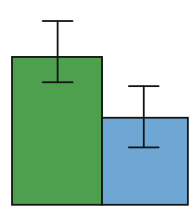

(3) transitif
Allemand

Chinois

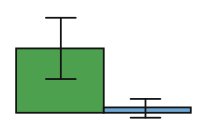

(4) réflechi

Verbes transitifs/intransitifs $(1,2)$ et transitifs/réfléchis $(3,4)$

Figure 12. Fréquence de production attendue de clitiques objets selon les conditions pour les deux groupes, chinois et allemand; les barres d'erreurs reflètent des intervalles de confiance $(95 \%)$

ainsi qu'entre le niveau en français et la production de clitiques attendus $(r=0,2 \circ$, $p<0$,oI). Également, une corrélation significative est trouvée entre la mémoire de travail et le niveau en français $\mathrm{L}_{2}(r=0,23, p<0,00 \mathrm{I})$. Les régressions linéaires mixtes, avec les deux prédicteurs combinés, montrent que le niveau en français va avoir des effets significatifs sur la production attendue de clitiques objets au-delà de la mémoire de travail (chisq $=4,7286, d f=\mathrm{I}, p<0,05)$. Cela étant, le score obtenu à l'«empan de lecture» a indépendamment des effets significatifs sur la production attendue de clitiques objets (chisq $=6,2559, d f=\mathrm{I}, p<0,05)$. Les autres facteurs se sont avérés n'avoir aucune influence significative sur la production attendue de clitiques objets.

\subsubsection{Corrélations pour les sujets allemands}

Des analyses semblables ${ }^{12}$ à celles réalisées pour les données des apprenants chinois ont été effectuées pour les apprenants allemands concernant la corrélation entre les réponses de clitiques objets corrects et les autres facteurs potentiels comme l'«empan de lecture», le niveau en français $\left(\mathrm{B}_{1} / \mathrm{B}_{2}, \mathrm{~B}_{2} / \mathrm{C}_{1}, \mathrm{C}_{1}, \mathrm{C}_{2}\right)$, l'année du début d'apprentissage, l'usage du français ou la date d'arrivée en France ${ }^{13}$. Aucune corrélation significative n'est trouvée entre la mémoire de travail et le niveau en français L2 $(r=0,09, \mathrm{~ns})$. Les régressions linéaires mixtes, avec les deux prédicteurs combinés, montrent que le niveau en français va avoir des effets significatifs sur la production

12. En raison d'un problème technique, un participant allemand a été exclu pour ces analyses, car nous n'avions pas ses données.

13. L'arrivée en France concerne les participants qui vivaient ici pendant l'expérience. Afin de pouvoir faire la comparaison avec ces participants-là, on a donc considéré que les participants qui vivaient en Allemagne avaient «o année» même s'ils ont pu vivre en France auparavant. 
attendue de clitiques objets au-delà de la mémoire de travail (chisq $=5,7342, d f=\mathrm{I}$, $p<0,05)$. En revanche, le score obtenu à l'«empan de lecture» ne va pas avoir indépendamment des effets significatifs sur la production attendue de clitiques objets (chisq $=\mathrm{I}, 4204, d f=\mathrm{I}, \mathrm{ns}$ ). Par ailleurs, des corrélations positives entre la production attendue de clitiques et le niveau en français $(r=0,28, p<0,00 \mathrm{I})$, l'usage du français $(r=0,23, p<0, \mathrm{OI})$ et les années passées en France $(r=0,29, p<0, \mathrm{OOI})$ sont constatées, et également entre la production attendue de clitiques et la mémoire de travail $(r=0,17, p<0,05)$.

\section{Discussion}

Concernant les résultats, et notamment ceux plus performants pour le groupe allemand, il faut rappeler que les participants allemands ont commencé à apprendre le français plus tôt par rapport aux participants chinois. Cette différence entre les deux groupes nécessite donc de considérer les résultats avec une certaine prudence. La production de pronoms clitiques objets semble bien représenter une réelle difficulté pour les apprenants en français L2 comme l'a montré l'expérience avec les apprenants chinois et les apprenants allemands, notamment en ce qui concerne les niveaux débutants et intermédiaires. Cela étant, à un niveau très avancé et proche de celui d'un locuteur natif comme les niveaux $\mathrm{C}_{\mathrm{I}}$ et $\mathrm{C}_{2}$, cette catégorie fonctionnelle semble être maitrisée et les tendances de production ressemblent beaucoup à celles observées en français LI, notamment chez les apprenants allemands qui ont un niveau plus avancé. Les résultats en L2 montrent un pattern semblable à celui des résultats en $\mathrm{Lr}$ en étant bien évidemment moins élevés. Notre expérience semble alors confirmer une acquisition en L2 similaire à la Li, soit par un accès à une "grammaire universelle» avec une refixation des paramètres soit par l'usage et l'entraînement.

Nous constatons également que les apprenants chinois et allemands recourent aux SNs objets à la place des clitiques plus aisément que les participants français. Concernant la production de SNs objets, un relecteur a proposé comme explication un effet d'amorçage syntaxique (priming). Certes, le contexte favorisant les conditions contenait pour les clitiques un SN objet («Le dragon boit l'eau de la rivière», encadré I), ce qui pourrait provoquer un effet d'amorçage, d'où la reprise du SN objet dans les réponses des participants. Cependant, cela concernerait uniquement les groupes allemand et chinois puisque le groupe français n'a pratiquement pas produit de SNs objets et la différence de production de SNs objets entre les apprenants chinois et allemands et les locuteurs natifs du français n'est pour autant pas significative. En outre, le fait que la condition (2) intransitif soit très bien réussie dans les deux groupes montre que les sujets n'utilisent pas les clitiques par hasard sinon nous devrions les trouver dans cette condition de manière bien plus importante.

Concernant les raisons de ces difficultés d'acquisition constatées au début de l'apprentissage, l'expérience montre que le niveau en français a effectivement un effet significatif et que moins les connaissances en français étaient élevées, plus la production de pronoms clitiques était basse. 
L'hypothèse (a) de la «structure de surface» de Clahsen et Felser (2006a et b) est en partie en accord avec nos résultats si nous nous appuyons sur la distance structurale puisque dans ce cas, les apprenants devraient avoir des difficultés à produire des clitiques objets. Cependant, si nous tenons compte plutôt de la distance linéaire, cette hypothèse ne peut pas expliquer nos résultats du fait que les clitiques objets ne devraient poser aucune difficulté aux apprenants, ce qui n'est pas le cas dans notre expérience. Par ailleurs, même en tenant compte de la distance structurale, les participants devraient avoir toujours autant de difficultés pour maitriser cette catégorie fonctionnelle même en progressant dans la L2, ce qui ne semble pas être le cas puisque nous voyons que les apprenants à un niveau avancé se rapprochent des natifs en ce qui concerne leur production de clitiques.

L'hypothèse (b) qui concerne l'influence de la Li (White, 1996) ne semble pas refléter entièrement les résultats, puisque l'on constate des productions similaires pour les deux groupes chinois et allemand alors que la Li devrait montrer des disparités dans les productions en raison d'une grande différence entre la langue allemande et le chinois. La Li ne semble donc pas être un facteur prédominant et exclusif pour nos résultats.

L'hypothèse (c) sur les interfaces (Sorace et Filiaci, 2006) pourrait expliquer en partie les résultats, notamment les écarts entre les niveaux, surtout si l'on considère les clitiques comme relevant de l'interface pragmatique. Cependant, il est difficile de définir son impact en l'absence d'autres facteurs manipulés pour vérifier clairement le rôle des interfaces.

L'hypothèse (d) sur la mémoire de travail (Grüter et Crago, 20I2) semble en accord avec nos résultats, spécialement pour le groupe chinois puisque l'on constate que la mémoire de travail joue un rôle important dans la performance de production des clitiques, rôle qui va au-delà de celui joué par le niveau en français. Aucune corrélation n'a été trouvée entre le test de mémoire et la production attendue de clitiques indépendamment du niveau de français L2 pour les sujets allemands, certes, mais il est nécessaire de rappeler que ces derniers avaient un niveau plus élevé en français que les sujets chinois, ce qui supposerait sûrement que leur production en français L2 pouvait ne pas exiger autant d'efforts pour la mémoire de travail, d'où l'absence d'effet. La mémoire de travail semble jouer un rôle important au début de l'apprentissage, mais ce rôle tend à diminuer lorsque le participant arrive à un niveau proche de celui du natif car cela devient effectivement plus facile, comme nous avons pu le voir avec le groupe allemand. En outre, les années passées en France et l'usage quotidien du français semblent toujours avoir un impact sur la production en français L2 puisque nous trouvons une corrélation significative avec la production attendue de clitiques chez les participants allemands.

Nos résultats viennent compléter alors l'étude menée par Grüter et Crago (20I2) soutenant la théorie de problèmes de mémoire de travail en tant qu'explication des difficultés d'acquisition des clitiques. Cela est également en lien alors avec l'hypothèse de complexité computationnelle proposée par Jakubowicz et al. (1998). Afin de 
confirmer plus solidement ces théories, il serait intéressant de tester l'«empan de lecture» avec des participants natifs du français afin de voir si le rôle de la mémoire de travail joue en Li pour cette catégorie syntaxique, ce qui n'a pas été fait dans cette expérience. Cela permettrait aussi de faire le lien avec l'omission des clitiques objets par les locuteurs natifs du français.

Un autre résultat à prendre en compte est, en outre, la non-production des clitiques objets par les apprenants chinois et allemands, mais également par les participants français. Comme cela a été remarqué dans l'expérience, les apprenants français ne produisent pas systématiquement de clitiques, notamment dans la condition (I). En fait, le phénomène de l'omission des pronoms clitiques objets, plus généralement des SNs objets, chez l'adulte francophone, avait déjà été constaté dans d'autres études (entre autres, Pirvulescu, 2006; Belzil, 2009; Rasetti, 2003; Goldberg, 200I). La production de clitiques objets avait déjà été remarquée comme non catégorique contrairement aux pronoms clitiques sujets, puisque selon le contexte linguistique ou extralinguistique, la production de l'objet n'était pas systématique pour les verbes transitifs. La production de l'objet peut en fait être optionnelle comme en [19] (Belzil, 2009).

[ig] a. Paul mange pro.

b. Paul mange la pizza ${ }_{i}$

À ce propos, Belzil (2009: 68) fait remarquer que cette non-réalisation ne serait pas imputable à certains types de verbes mais pourrait être étendue à l'ensemble des verbes. Cette omission du clitique, notamment en français Li dans les verbes de type transitif/intransitif, peut s'expliquer par l'approche syntaxique de Cummins et Roberge (2005) qui va s'appuyer également sur la sémantique et la pragmatique. De manière générale, ces auteurs considèrent qu'il n'y aurait pas de véritables différences entre une phrase avec un clitique objet et la même phrase sans ce dernier, mis à part sur le plan phonologique (exemple [ig]). Selon eux, le verbe demande syntaxiquement toujours un complément d'objet, ce qu'ils appellent l'hypothèse de la «nécessité de la transitivité» (Transitivity Requirement). Le verbe et l'objet seraient aussi sémantiquement dans «une relation hyponimique» (Hale et Keyser, 2002), ce qui fait que l'objet serait interprété selon la sémantique du verbe. Cela signifierait que le SN objet serait alors sélectionné par le verbe comme le montre la figure I3 (Cummins et Roberge, 2005).

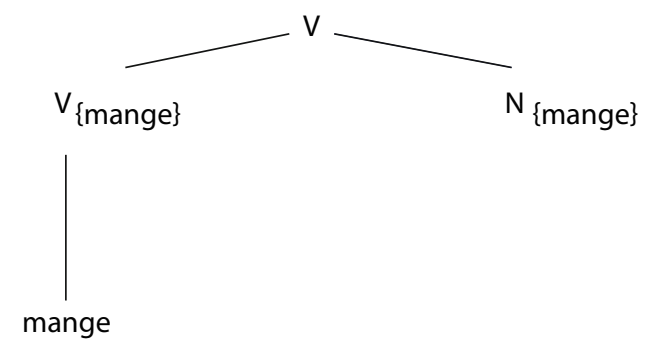

Figure 13. Illustration de la relation hyponimique (inspirée de Hale et Keyser, 2002) 
Ainsi, pour identifier l'élément pro et le mettre en lien avec l'antécédent comme en figure $\mathrm{I}_{3}$, le locuteur s'aiderait alors de manière pragmatique du principe I de Levinson qui s'appuie sur la «maxime informative» (Maxim of Informativeness) de Grice (1975) (exemple [20]). Cette dernière stipule que l'interlocuteur fait un lien entre l'information linguistique fournie par le locuteur et le contexte linguistique ou extralinguistique présent. Selon ce contexte, l'objet pourrait donc plus facilement être omis car le lien est facilement identifiable. Ceci conduirait alors à la non-production de clitiques sans que le sens en soit altéré, puisque le verbe est par nature transitif et l'objet est déjà compris dans la syntaxe et la sémantique du verbe.

[20] Principe I (Levinson, 2000: II4, in Cummins et Roberge, 2005: IO-II)

Speaker's maxim: the maxim of Minimization: "Say as little as necessary"; that is, produce the minimal linguistic information sufficient to achieve your communicational ends...

Recipient's corollary: the Enrichment Rule. Amplify the informational content of the speaker's utterance, by finding the most specific interpretation... Specifically:

... Assume that stereotypical relations obtain between referents and events...

... Avoid interpretations that multiply entities referred to (assume referential parsimony): specifically, prefer coreferential readings of reduced NPs (pronouns or zeros)...

Ceci pourrait permettre de comprendre l'omission dans notre expérience des clitiques objets dans les phrases avec des verbes à conditions (I) et (2), soit de type transitif/intransitif, d'autant plus que les clitiques objets semblent être une catégorie également difficile pour les francophones, comme l'indique Mougeon (1995:66): «Les pronoms clitiques objet sont incontestablement une pierre d'achoppement pour les locuteurs qui sous-utilisent le français [...]. Le fait que r'on puisse les récupérer avec le contexte est sans doute propice à leur évitement». Cette hypothèse pourrait également expliquer pourquoi la production de clitiques objets est significativement plus importante dans la condition (3) transitif pour les verbes transitifs/réfléchis. En effet, dans ce contexte, l'omission est moins propice puisqu'il n'y a pas la condition (2) impliquant la possibilité tout à fait grammaticale de ne pas produire le clitique. La condition (4) ne permet pas nécessairement au niveau grammatical la production du verbe sans le clitique et il serait difficile d'identifier l'élément pro et de faire le lien avec l'antécédent pour le réfléchi d'un point de vue pragmatique en français Li. Pour ce type de verbes, les francophones vont donc préférer recourir aux clitiques de façon plus fréquente afin que leur énoncé soit selon eux jugé correct et compréhensible. On pourrait alors penser que l'omission des clitiques objets en L2 serait finalement à mettre en relation avec certains types de verbes, soit ceux qui privilégient les conditions transitif et intransitif. Nous avons par ailleurs observé une différence significative entre les deux types de verbes transitifs/intransitifs et transitifs/réfléchis en français L2. Dans le groupe français, la production de clitiques réfléchis est numériquement moins élevée que celle de clitiques objets en condition (3), ce qui l'est encore moins dans le groupe chinois et le groupe allemand, avec une différence significative. Cette catégorie syntaxique pourrait en fait être alors plus difficile à 
traiter. Il faut également noter que le groupe allemand réussit significativement mieux à produire les réfléchis que le groupe chinois, probablement en raison d'une plus grande ressemblance avec la structure du réfléchi en allemand par rapport au chinois. Il serait alors pertinent de comprendre l'origine de ce problème, notamment si cela était aussi en lien avec les capacités de mémoire. Dans l'expérience, les questions amenant des pronoms clitiques objets comme le ou la étaient toujours contextualisées (qu'est-ce qu'il fait avec?) alors que les questions conduisant à la production de pronoms réfléchis ne l'étaient pas (qu'est-ce qu'il fait?). Le fait de ne pas avoir une référence au réfléchi dans la situation de communication serait-il un facteur expliquant la difficulté de production de cette catégorie notamment en L2 puisqu'il faudrait le récupérer d'un point de vue pragmatique? Ceci serait plus en lien avec l'hypothèse des interfaces. Afin de clarifier cela, des études restent à faire avec des langues aux propriétés semblables à celles du français en L2 comme en Li.

Ainsi, si l'on reprend nos quatre hypothèses de départ, la difficulté de produire les clitiques n'a pas pu être éclaircie totalement par un problème d'interface ni par l'hypothèse de la «structure de surface» dans le cadre de cette expérience (même si les résultats sont généralement compatibles avec certaines versions de ces hypothèses). Finalement, l'acquisition des clitiques en L2 semble, dans notre contexte, être certainement facilitée par la mémoire de travail. De fait, au travers de nos résultats, l'acquisition en L2 se rapproche de l'acquisition en LI avec pour facteur dominant un problème de mémoire de travail, ce qui laisse suggérer que les apprenants en L2 peuvent se rapprocher du niveau des natifs en ce qui concerne l'acquisition des pronoms clitiques.

\section{Remerciements}

Nous tenons à remercier l'Institut français de Berlin pour nous avoir généreusement laissé passer des expériences dans leurs locaux.

Nous tenons également à remercier nos relecteurs pour leurs commentaires et leurs suggestions qui nous ont permis d'approfondir la présentation de nos recherches.

\section{Références}

Baayen, R. H., Davidson, D. J. et Bates, D. M. 2008. Mixed-Effects Modeling with Crossed Random Effects for Subjects and Items. Journal of Memory and Language $59(4): 390-412$.

BeLzIL, I. 2009. L'asymétrie entre l'acquisition des clitiques sujets/objets chez les enfants francophones et l'optionalité dans la grammaire enfantine. Thèse de doctorat. Université de Toronto.

Chiv, B. 1995. An Object Clitic Projection in Mandarin Chinese. Journal of East Asian Linguistics 4 (2): 77-II7. 
Clahsen, H. et Felser, C. 2006a. How Native-Like Is Non-native Language Processing? Trends in Cognitive Sciences Io (I2): 564-570.

Clahsen, H. et Felser, C. 2006b. Continuity and Shallow Structures in Language Processing. Applied Psycholinguistics 27 (I): I07-I26.

Cummins, S. et Roberge, Y. 2005. A Modular Account of Null Objects in French. Syntax 8 (I) : 44-64.

Cuza, A., Pérez-Leroux, A. T. et Sánchez, L. 2013. The Role of Semantic Transfer in Clitic Drop among Simultaneous and Sequential Chinese-Spanish Bilinguals. Studies in Second Language Acquisition 35 (I) : 93-I25.

Daneman, M. et Carpenter, P. A. I980. Individual Differences in Working Memory and Reading. Journal of Verbal Learning and Verbal Bebavior i9 (4): 450-466.

Daneman, M. et Hannon, B. 2007. What do Working Memory Span Tasks Like Reading Span Really Measure? In N. Osaka, R. H. Logie et M. D'Esposito (éd.), The Cognitive Neuroscience of Working Memory. Oxford: Oxford University Press : 2I-42.

Darobbers, R. et XIAo Planes, X. 1998. Éléments fondamentaux de la phrase chinoise. Paris : Éditions You-Feng.

EIsEnbeIss, S. 20Io. Production Methods in Language Acquisition Research. In E. Blom et S. Unsworth (éd.), Experimental Methods in Language Acquisition Research. Philadelphie: J. Benjamins : II-34.

Ferreira, F. 2000. Syntax in Language Production: An Approach Using Tree-Adjoining Grammars. In L. WheEldon (éd.), Aspects of Language Production. Hove: Psychology Press : 29I-330.

FERreira, F. 2003. The Misinterpretation of Noncanonical Sentences. Cognitive Psychology 47 (2): $164-203$.

Ferreira, F., Bailey, K. G. D. et Ferraro, V. 2002. Good-Enough Representations in Language Comprehension. Current Directions in Psychological Science II (I) : II-I5.

Goldberg, A. 20or. Patient Arguments of Causative Verbs Can Be Omitted: The Role of Information Structure in Argument Distribution. Language Sciences 23 (4-5) : 503-524.

Grice, H. P. I975. Logic and Conversation. In P. Cole et J. Morgan (éd.), Syntax and Semantics. New York - Londres: Academic Press. Vol. 3: Speech Acts: 4I-58.

GrüTER, T. 2006. Object Clitics and Null Objects in the Acquisition of French. Thèse de doctorat. McGill University.

Grüter, T. et Crago, M. 20I2. Object Clitics and Their Omission in Child L2 French: The Contributions of Processing Limitations and LI Transfer. Bilingualism: Language and Cognition I5 (3): 53I-549.

Hale, K. et Keyser, S. J. 2002. Prolegomenon to a Theory of Argument Structure. Cambridge Londres: MIT Press.

Hamann, C., Rizzi, L. et Frauenfelder, U. 1996. On the Acquisition of Subject and Object Clitics in French. In H. Clahsen (éd.), Generative Perspectives on Language Acquisition: Empirical Findings, Theoretical Considerations, and Crosslinguistic Comparisons. Language Acquisition and Language Disorders I4. Amsterdam - Philadelphie: J. Benjamins: 309-334. 
Hernandez, A., Li, P. et MacWhinney, B. 2005. The Emergence of Competing Modules in Bilingualism. Trends in Cognitive Sciences 9 (5): 220-225.

Huang, C.-T. J., LI, Y.-H. A. et LI, Y. 2009. The Syntax of Chinese. Cambridge Syntax Guides. Cambridge: Cambridge University Press.

Jakubowicz, C., Nash, L., Rigaut, C. et Gérard, C.-L. 1998. Determiners and Clitic Pronouns in French-Speaking Children with SLI. Language Acquisition 7 (2-4) : I13-I60.

Jakubowicz, C. et Rigaut, C. 2000. L'acquisition des clitiques nominatifs et des clitiques objets en français. Revue canadienne de linguistique 45: II9-I57.

Joshi, A. K., Levy, L. S. et Takahashi, M. 1975. Tree Adjunct Grammars. Journal of Computer and System Sciences Io (I): 136-163.

Levinson, S. C. 200o. Presumptive Meanings: The Theory of Generalized Conversational Implicature. Cambridge - Londres: MIT Press.

Li, P., Zhang, F., Tsai, E. et Puls, B. 20I3. Language History Questionnaire (LHQ 2.o) : A New Dynamic Web-Based Research Tool. Bilingualism: Language and Cognition $17(3): 673-68$ o.

Miller, P. H. 1992. Clitics and Constituents in Phrase Structure Grammar. New York - Londres: Garland.

Miller, P. H. et Monachesi, P. 2003. Les pronoms clitiques dans les langues romanes. In D. GodARD (éd.), Les langues romanes: problèmes de la phrase simple. Paris: CNRS Éditions: 67-I23.

Mougeon, R. 1995. Diversité sociolinguistique au sein d'une communauté francophone minoritaire: les Franco-Ontariens. Linx 33: 47-69.

Pirvulescu, M. 2006. Theoretical Implications of Object Clitic Omission in Early French : Spontaneous vs. Elicited Production. Catalan Journal of Linguistics 5: 22I-236.

Prévost, P. 2009. The Acquisition of French: The Development of Inflectional Morphology and Syntax in LI Acquisition, Bilingualism, and L2 Acquisition. Language Acquisition and Language Disorders $5 \mathrm{I}$. Amsterdam - Philadelphie: J. Benjamins.

Rasetti, L. 2003. Optional Categories in Early French Syntax: A Developmental Study of Root Infinitives and Null Arguments. Thèse de doctorat. Université de Genève.

Riegel, M., Pellat, J.-C. et Rioul, R. 2009. Grammaire méthodique du français. Paris: PUF.

Rossi, E., Kroll, J. F. et Dussias, P. E. 20I4. Clitic Pronouns Reveal the Time Course of Processing Gender and Number in a Second Language. Neuropsychologia 62: II-25.

SELINKER, L. 1972. Interlanguage. International Review of Applied Linguistics in Language Teacbing - IRAL Io (I-4): 209-232.

Sneed German, E., Herschensohn, J. et Frenck-Mestre, C. 20i5. Pronoun Processing in Anglophone Late L2 Learners of French: Behavorial and ERP Evidence. Journal of Neurolinguistics 34: 15-40.

Sorace, A. 2orr. Pinning Down the Concept of «Interface» in Bilingualism. Linguistic Approaches to Bilingualism I (I): $\mathrm{I}-33$.

Sorace, A. et Filiaci, F. 2006. Anaphora Resolution in Near-Native Speakers of Italian. Second Language Research 22 (3):339-368. 
Schwartz, B. D. et Sprouse, R. A. 1994. Word Order and Nominative Case in Non-native Language Acquisition. In T. HoEkstra et B. D. SchwArtz (éd.), Language Acquisition Studies in Generative Grammar. Language Acquisition and Language Disorders 8. Amsterdam - Philadelphie: J. Benjamins: 317-368.

Schwartz, B. D. et Sprouse, R. A. 1996. L2 Cognitive States and the Full Transfer/Full Access Model. Second Language Research I2 (I) : 40-72.

ŠTícHA, F. 20I5. Wie identifiziert man das lexikalisch unausgedrückte Objekt im Satz? Article en ligne à l'adresse suivante: http://hypermedia.ids-mannheim.de/evalbu/ festschrift/sticha.pdf.

Towell, R. et Hawnins, R. 1994. Approaches to Second Language Acquisition. Clevedon: Multinligual Matters.

TsedryK, K. et PunKo, I. 2008a. Pronoms clitiques accusatifs et datifs chez les enfants francophones. In S. JonEs (éd.), Actes du congrès annuel de l'Association canadienne de linguistique. En ligne à l'adresse suivante: http://homes.chass.utoronto.ca/ cla-acl/ actes2008/CLA2008_Tsedryk_Punko.pdf.

TsEDryK, K. et Punko, I. 2008b. L'acquisition des pronoms clitiques en français langue seconde (L2). In J. Durand, B. Habert et B. Laks (éd.), Congrès mondial de linguistique française (CMLF 2008). Paris: Institut de linguistique française: 1859-I870. En ligne à l'adresse suivante: http://www.linguistiquefrancaise.org/articles/cmlf/pdf/2008/oI/ cmlfo8026.pdf.

Vainikka, A. et Young-Scholten, M. 1994. Direct Access to X'-theory: Evidence from Korean and Turkish Adults Learning German. In T. Hoekstra et B. D. Schwartz (éd.), Language Acquisition Studies in Generative Grammar. Language Acquisition and Language Disorders 8. Amsterdam - Philadelphie: J. Benjamins : 265-316.

VAinikKa, A. et Young-Scholten, M. 1996. Gradual Development of L2 Phrase Structure. Second Language Research I2 (I) : 7-39.

VAN DER VELDE, M. 2003. Déterminants et pronoms en néerlandais et en français: syntaxe et acquisition. Thèse de doctorat. Université Vincennes-Saint-Denis - Paris 8.

Vinet, M.-T. et Zhou, H. 2003. La possession inaliénable en chinois mandarin et en français. Cabiers de linguistique - Asie orientale 32 (2): 157-193.

White, L. 1996. Clitics in L2 French. In H. Clahsen (éd.), Generative Perspectives on Language Acquisition: Empirical Findings, Theoretical Considerations, and Crosslinguistic Comparisons. Language Acquisition and Language Disorders 14. Amsterdam Philadelphie: J. Benjamins: $335-368$.

White, L. 2003. Second Language Acquisition and Universal Grammar. Cambridge: Cambridge University Press.

$\mathrm{Xu}, \mathrm{D}$. 20Io. Initiation à la syntaxe chinoise: des repères et des analyses pour écrire et parler juste. Collection Langues-INALCO. Paris: L'Asiathèque. 


\section{Annexe 1. Liste des items de l'expérience de production élicitée}

\section{Verbes à conditions (1) transitif et (2) intransitif}

1. Boire

\section{Transitif}

Le dragon et le crocodile sont tous les deux près de la rivière. Cependant, le crocodile veut aller de l'autre côté pour voir ses autres amis. Du coup, le dragon boit l'eau de la rivière afin que son ami le crocodile puisse passer. Il y a beaucoup d'eau dans la rivière car il a beaucoup plu ces derniers temps. Qu'est-ce que fait le dragon avec l'eau de la rivière? (boire)

\section{Intransitif}

Le dragon et le crocodile sont tous les deux près de la rivière. Cependant, le crocodile veut aller de l'autre côté pour voir ses autres amis. Mais le dragon ne veut pas bouger car il a très soif. Du coup, il boit et se sent bien mieux. Le crocodile rejoint pendant ce temps ses amis de l'autre côté de la rivière. Il y a beaucoup d'eau dans la rivière car il a beaucoup plu ces derniers temps. Qu'est-ce que fait le dragon? (boire)

\section{Descendre}

\section{Transitif}

Le perroquet et le pingouin se connaissent depuis longtemps. Un jour, le perroquet demande de l'aide à son ami avec un rocher dont il a besoin. Le pingouin accepte sans hésitation. Du coup, le pingouin descend le rocher dans la grotte du perroquet. La grotte est sombre mais il y a un vent très agréable. Qu'est-ce que fait le pingouin avec le rocher? (descendre)

\section{Intransitif}

Le perroquet et le pingouin se connaissent depuis longtemps. Un jour, le pingouin et le perroquet déjeunent tous les deux à côté d'un rocher. Le pingouin veut ensuite faire une sieste. Pour cela, il descend rapidement dans sa grotte. La grotte est sombre mais il y a un vent très agréable.

Qu'est-ce que fait le pingouin? (descendre)

3. Fuir

\section{Transitif}

La coccinelle et le lion se retrouvent par basard près de l'arbre. La coccinelle n'aime pas le lion et a peur de lui. Du coup, quand elle arrive près de l'arbre, la coccinelle fuit le lion. L'arbre n'a pas encore de fruits car le printemps n'est toujours pas arrivé.

Qu'est-ce que fait la coccinelle avec le lion? (fuir)

\section{Intransitif}

La coccinelle et le lion se retrouvent par hasard près de l'arbre. Le lion monte dans l'arbre et s'endort. Cependant, il ronfle et fait beaucoup de bruit. Du coup, la coccinelle, énervée, fuit pour trouver un endroit plus calme. L'arbre n'a pas encore de fruits car le printemps n'est toujours pas arrivé. Qu'est-ce que fait la coccinelle? (fuir) 


\section{Transitif}

La chenille et l'escargot se disputent car chacun veut la feuille. Finalement, la chenille gagne. Elle mange la feuille car elle a très faim. L'escargot est triste et pleure beaucoup.

Qu'est-ce que fait la chenille avec la feuille? (manger)

\section{Intransitif}

La chenille et l'escargot jouent ensemble à des courses de vitesse avec une feuille sur le dos. Comme la chenille est plus forte, elle gagne toutes les récompenses. À la fin, la chenille est très fatiguée et affamée et donc, elle mange. L'escargot est triste et pleure beaucoup. Qu'est-ce que fait la chenille? (manger)

\section{Monter}

\section{Transitif}

Le zèbre et la girafe ont décidé de faire un pique-nique au sommet de la montagne. Aujourd'bui, c'est la girafe qui s'occupe du pique-nique. Le zèbre attend au sommet de la montagne. Pendant ce temps, la girafe monte le pique-nique jusqu'à l'endroit où se trouve le zèbre. La montagne est vraiment haute et le sommet se trouve au milieu des nuages.

Qu'est-ce que fait la girafe avec le pique-nique? (monter)

\section{Intransitif}

Le zèbre et la girafe ont décidé de faire un pique-nique au sommet de la montagne. Le zèbre a tout préparé et attend la girafe au sommet de la montagne. Alors, la girafe monte rapidement pour rejoindre le zèbre. La montagne est vraiment baute et le sommet se trouve au milieu des nuages. Qu'est-ce que fait la girafe? (monter)

\section{Cuisiner}

\section{Transitif}

La grenouille et la girafe déjeunent ensemble tous les mercredis. Aujourd'bui, la girafe a apporté des algues pour le repas. Ainsi, la grenouille cuisine les algues et pendant ce temps, la girafe dresse la table. La grenouille et la girafe sont de bonnes amies depuis très longtemps.

Qu'est-ce que fait la grenouille avec les algues? (cuisiner)

\section{Intransitif}

La grenouille et la girafe déjeunent ensemble tous les mercredis. Aujourd'bui, le repas a lieu chez la grenouille qui habite dans la rivière où l'on peut trouver beaucoup d'algues. La grenouille cuisine pendant que la girafe dresse la table. La grenouille et la girafe sont de bonnes amies depuis très longtemps.

Qu'est-ce que fait la grenouille? (cuisiner) 


\section{Transitif}

Le mouton babite dans une jolie petite maison assez loin de celle du zèbre. Un jour, le mouton doit s'absenter pour rendre visite à sa famille. Du coup, le zèbre garde la maison du mouton en sécurité. Comme il s'ennuie, le zèbre broute l'herbe à côté de la maison du mouton. Le zèbre aime bien passer du temps avec ses amis et les aider en cas de besoin.

Qu'est-ce que fait le zèbre avec l'herbe? (brouter)

\section{Intransitif}

Le mouton habite dans une jolie petite maison assez loin de celle du zèbre. Un jour, le mouton part s'amuser dans un champ où il y a de l'berbe. Cependant, le zèbre est paresseux et reste chez lui. Comme il a faim, le zèbre broute. Le zèbre aime bien passer du temps avec ses amis et les aider en cas de besoin. Qu'est-ce que fait le zèbre? (brouter)

8. Rentrer

\section{Transitif}

La vache et la chenille se promènent ensemble sous un très beau temps. Ensuite, comme elle voit une feuille verte appétissante, la chenille rentre la feuille dans son frigo pour la garder au frais. Son amie la vache aime marcher dans les prairies pendant de longs moments.

Qu'est-ce que fait la chenille avec la feuille? (rentrer)

\section{Intransitif}

La vache et la chenille se promènent ensemble sous un très beau temps. Cependant, la chenille est très vite fatiguée car elle a beaucoup travaillé ces derniers temps. Du coup, la chenille rentre chez elle rapidement. Son amie la vache aime marcher dans les prairies pendant de longs moments.

Qu'est-ce que fait la chenille? (rentrer)

\section{Verbes à conditions (3) transitif et (4) réfléchi}

1. Baisser

\footnotetext{
Transitif

Le singe et le chat sont voisins. Le singe veut faire une farce au chat. Ainsi, il réussit à faire entrer le chat dans le carton puis baisse les côtés du carton pour enfermer le chat. Le chat ne comprend pas ce qu'il se passe et a très peur.

Qu'est-ce que fait le singe avec les côtés du carton? (baisser)

\section{Réfléchi}

Le singe et le chat sont voisins. Le singe veut faire une farce au chat en se cachant dans le carton. Le chat ne retrouve plus le singe et s'inquiète. Du coup, le chat baisse sa tête pour voir ce qu'il y a dans le carton et aperçoit le singe.

Qu'est-ce que fait le chat pour voir ce qu'il y a dans le carton? (baisser)
} 
2.

\section{Transitif}

L'ours invite son amie la chèvre dans sa maison couverte de paille. La chèvre arrive mais à cause $d u$ vent, ses poils sont complètement en désordre et elle ne voit plus rien. Alors, l'ours brosse la chèvre et celle-ci est de nouveau coiffée. La maison comporte beaucoup de pièces et le salon est très grand.

Qu'est-ce que fait l'ours avec la chèvre? (brosser)

\section{Réfléchi}

L'ours invite son amie la chèvre dans sa maison couverte de paille. La chèvre arrive trop tôt et l'ours n'est pas encore coiffé, tous ses poils sont en désordre. La chèvre attend alors dans le salon et pendant ce temps, l'ours brosse ses poils. La maison comporte beaucoup de pièces et le salon est très grand.

Qu'est-ce que fait l'ours? (brosser)

\section{Cacher}

\section{Transitif}

Le renard et le poisson se voient régulièrement pour passer du temps ensemble. Un jour, la famille $d u$ poisson cherche celui-ci, mais le poisson ne veut pas la voir. Pour l'aider, le renard cache le poisson derrière la feuille. En cette saison, les feuilles sont très colorées et le temps est magnifique. Qu'est-ce que fait le renard avec le poisson? (cacher)

\section{Réfléchi}

Le renard et le poisson se voient régulièrement pour passer du temps ensemble. Aujourd'bui, le renard veut faire une farce au poisson. Du coup, il cache son corps derrière la feuille. En arrivant, le poisson ne voit que la tête du renard et a très peur. En cette saison, les feuilles sont très colorées et le temps est magnifique.

Qu'est-ce que fait le renard? (cacher)

\section{Gratter}

\section{Transitif}

Le tigre et le panda sont ensemble près de la rivière alors qu'il fait un très beau temps. Le panda gratte le tigre à côté de la rivière, parce que le tigre a mal au dos à cause de la chaleur. La rivière a des reflets dorés et argentés $d u$ fait $d u$ soleil.

Qu'est-ce que fait le panda avec le tigre? (gratter)

\section{Réfléchi}

Le tigre et le panda sont ensemble près de la rivière alors qu'il fait un très beau temps. Le panda gratte son dos à côté de la rivière alors que le tigre dort dans un champ de l'autre côté de la rivière. $L$ a rivière a des reflets dorés et argentés $d u$ fait $d u$ soleil. Qu'est-ce que fait le panda? (gratter) 


\section{Transitif}

Le phoque et la baleine ne sont pas de bons amis mais babitent tous les deux au milieu des algues. Comme il n'est pas content, le phoque blesse la baleine, car elle a pris toute la place au milieu des algues. Les algues sont très nombreuses et de différentes sortes.

Qu'est-ce que fait le phoque avec la baleine? (blesser)

\section{Réfléchi}

Le phoque et la baleine ne sont pas de bons amis mais habitent tous les deux au milieu des algues. La baleine est restée dans sa maison, alors que le phoque se promène. Cependant, il est maladroit, il blesse son bras et a très mal. Les algues sont très nombreuses et de différentes sortes.

Qu'est-ce que fait le phoque? (blesser)

\section{Admirer}

\section{Transitif}

La souris et le canard se rencontrent souvent près de la rivière. La souris n'aime pas trop parler avec les autres animaux. Du coup, quand elle arrive près de la rivière, la souris admire juste le canard. La rivière n'est pas très grande mais très profonde.

Qu'est-ce que fait la souris avec le canard? (admirer)

\section{Réfléchi}

La souris et le canard se rencontrent souvent près de la rivière. La souris n'aime pas trop parler avec les autres animaux. Du coup, quand elle arrive près de la rivière, la souris admire seulement le reflet de son visage dans l'eau. La rivière n'est pas très grande mais très profonde. Qu'est-ce que fait la souris? (admirer)

\section{Pencher}

\section{Transitif}

L'araignée vit tranquillement dans l'herbe. Cependant, le cheval passe souvent à côté de l'araignée. Avec ses gros sabots, il penche l'herbe près de la maison de l'araignée. Cela dérange beaucoup l'araignée qui n'est pas contente du tout. Il fait toujours très beau à cet endroit.

Qu'est-ce que fait le cheval avec l'berbe? (pencher)

\section{Réfléchi}

L'araignée vit tranquillement dans l'herbe. Cependant, le cheval passe souvent à côté de l'araignée après s'être baigné dans la rivière. Puis, il penche son corps près de la maison de l'araignée pour observer l'araignée. Cela dérange beaucoup l'araignée qui n'est pas contente du tout. Il fait toujours très beau à cet endroit.

Qu'est-ce que fait le cheval? (pencher) 
8

\section{Transitif}

Le singe et le cochon sont de vieux amis. Aujourd'bui, ils se retrouvent à côté d'un carton. Cependant, le singe est le premier arrivé au lieu de rendez-vous. Puis, le cochon arrive. Pour punir le cochon de son retard, le singe enferme le cochon dans le carton pendant une petite minute. Le carton est solide mais n'est pas très joli et pas très grand non plus.

Qu'est-ce que fait le singe avec le cochon? (enfermer)

\section{Réfléchi}

Le singe et le cochon sont de vieux amis. Aujourd'hui, ils doivent se retrouver à côté d'un carton. Cependant, le singe est le premier arrivé au lieu de rendez-vous. Du coup, il décide de faire une farce au cochon. Pour cela, il enferme son corps dans le carton. Le cochon arrive, cherche et finit par trouver le singe dans le carton. Le carton est solide mais n'est pas très joli et pas très grand non plus.

Qu'est-ce que fait le singe? (enfermer)

\section{Verbes distracteurs}

1. Aboyer

Le cbien n'aime pas du tout le cochon. Cependant, tous les deux babitent dans la ferme et donc le chien le voit toujours. Mais comme il n'est pas content, le chien aboie dès qu'il voit le cochon. Le cocbon trouve cela très drôle.

Qu'est-ce que fait le chien? (aboyer)

2. Bouder

Le pingouin et le phoque babitent ensemble dans l'igloo. Aujourd'bui, le phoque est rentré très tard sans apporter quelque chose à manger. Du coup, le pingouin n'est pas content, alors il boude. Il y a toujours un grand soleil à l'endroit où babitent les deux animaux.

Qu'est-ce que fait le pingouin? (bouder)

\section{Courir}

Le cheval et le lion se retrouvent souvent en bas de la montagne pour se parler. Aujourd'bui, le cheval est en retard et du coup, il court le plus vite possible. Le lion attend l'arrivée de son ami pendant ce temps. La montagne est recouverte de neige en cette saison.

Qu'est-ce que fait le cheval? (courir) 
4.

Errer

La chenille habite dans une maison située au milieu de l'herbe. Comme il fait un très beau temps, elle invite l'escargot chez elle. Cependant, l'escargot ne trouve pas le chemin. Du coup, l'escargot erre dans l'herbe. La chenille part alors à sa rencontre. Il y a beaucoup d'herbe en cette saison. Qu'est-ce que fait l'escargot comme il ne trouve pas son chemin? (errer)

5. Éternuer

La pieuvre rend visite au poisson qui babite près des algues. Le poisson a attrapé un rbume et $d u$ coup, la pieuvre s'inquiète de sa santé. Le poisson éternue souvent tellement il est malade. La pieuvre décide donc de rester pour s'occuper de lui. Les algues sont nombreuses à l'endroit où babite le poisson. Qu'est-ce que fait le poisson? (éternuer)

6. Glisser

L'ours et le chien sont de bons amis qui habitent en bas de l'arbre. Un jour, pour trouver du miel pour son ami l'ours, le chien décide de grimper à l'arbre. Cependant, le chien est maladroit et glisse. L'ours ne peut s'empêcher de rire en le voyant. L'arbre n'a pas encore de fruits.

Qu'est-ce que fait le chien? (glisser)

7. Jouer

Le tigre aime rendre visite à son ami le dragon qui habite dans une grande maison rouge. Aujourd'bui, le tigre a pas mal de retard à cause de la pluie. Du coup, le dragon joue en l'attendant. La maison comporte plusieurs salles pour recevoir des amis et s'amuser tous ensemble.

Qu'est-ce que fait le dragon? (jouer)

\section{Marcher}

La coccinelle aime passer du temps avec son ami le chat près du lac. Tous les deux bavardent en se promenant sous un très beau temps. La coccinelle vole alors que le chat marche tranquillement. Le soleil ne va pas tarder à se coucher.

Qu'est-ce que fait le chat? (marcher)

\section{Nager}

Le cochon vient souvent près de la rivière pour boire de l'eau fraîche. Le crocodile babite à cet endroit mais apprécie la compagnie du cochon. Aujourd'bui, il fait chaud, donc le crocodile nage pour se rafraîcbir. Le cochon aime regarder les paysages près de la rivière.

Qu'est-ce que fait le crocodile? (nager) 
10. Naviguer

Le renard n'aime pas que les animaux passent par le pont, donc il surveille cet endroit. Comme le mouton doit passer, il prend un bateau. Ainsi, le mouton navigue sur l'eau et le renard ne peut rien faire pour l'en empêcher. Le renard n'est pas content.

Qu'est-ce que fait le mouton sur l'eau? (naviguer)

11. Partir

La girafe et la souris babitent ensemble dans la grotte et s'entendent assez bien. Mais un jour, la girafe s'énerve après la souris car elle n'a pas fait le ménage. Du coup, la girafe part. La souris est tout de même triste maintenant qu'elle babite seule dans la grotte.

Qu'est-ce que fait la girafe? (partir)

12. Patiner

Le lapin et le singe décident de partir en vacances dans un igloo. Comme il y a beaucoup de glace, le lapin patine avec élégance. Pendant ce temps, le singe surveille à ce que le lapin ne se fasse pas de mal. Il fait très chaud dans l'igloo.

Qu'est-ce que fait le lapin? (patiner)

\section{Pêcher}

La vache a invité le panda à passer l'après-midi près de la rivière. Comme il fait très chaud, la vache décide de se baigner dans l'eau. Pendant ce temps, le panda pèche. Il n'y a pas beaucoup de monde aujourd'bui près de la rivière.

Qu'est-ce que fait le panda? (pêcher)

\section{Pleurer}

L'araignée et le lapin font une compétition pour gagner la feuille. À la fin, l'araignée gagne baut la main et remporte la feuille en récompense. Le lapin est malbeureux et pleure beaucoup. En ce moment, les feuilles sont très rares à trouver.

Qu'est-ce que fait le lapin? (pleurer)

15. Ramper

La chenille babite dans le carton. Aujourd'bui, elle doit rendre visite à son ami le perroquet car il rentre de voyage. Ainsi, pour sortir de son carton, la chenille rampe avec agilité. Le carton est vieux mais très solide.

Qu'est-ce que fait la chenille pour sortir de son carton? (ramper) 
16. Rêver

Le chat babite tout en haut de la montagne. Aujourd'bui, le canard veut rejoindre le chat et découvrir sa maison. En arrivant en haut, le canard cherche son ami. En fait, le chat rêve en regardant les nuages. Par ce temps, les nuages sont nombreux et très jolis à regarder.

Qu'est-ce que fait le chat? (rêver)

17. $\quad$ Rire

Le cochon rend visite aujourd'bui au mouton dans sa petite maison rouge. Le mouton veut faire une farce et se cache derrière la porte. Quand le cochon entre, le mouton apparait derrière lui en faisant des grimaces. En voyant le mouton comme ça, le cochon rit beaucoup. La maison est solide et vient d'être construite.

Qu'est-ce que fait le cochon en voyant le mouton? (rire) 
Annexe 2. Modèle adapté du questionnaire d'histoire linguistique (Li et al., 2013)

PROFIL LINGUISTIQUE

(Ces informations resteront confidentielles.)

I. Âge :

2. Sexe:

3. Lieu de naissance, ville:

4. Profession et domaine:

5. Quelle(s) est/sont votre/vos langue(s) maternelle(s):

6. Quelle(s) langue(s) parliez-vous à la maison quand vous étiez petit(e):

7. Niveau d'études et discipline:

8. Quelle(s) autre(s) langue(s) parlez-vous?

- Langue étrangère $\mathrm{I}$ :

- Langue étrangère 2 :

- Langue étrangère 3:

9. Quel est votre niveau dans ce(s) langue(s) (I correspond à «débutant» et 5 à «avancé»):

- Langue étrangère $\mathrm{I}: \mathrm{I}$

$-2-3-4-5$

- Langue étrangère $2: \mathrm{I} \_-2-3 \_4-5$

- Langue étrangère $3: \mathrm{I}{ }_{-}^{2}-3 \_4-5$

Io. À quel âge avez-vous commencé à apprendre ce(s) langue(s)?

- Langue étrangère $\mathrm{I}$ :

- Langue étrangère 2 :

- Langue étrangère 3 :

II. Quel est l'usage par jour de ces langues pour les activités suivantes (/heure)?

\begin{tabular}{|l|l|l|l|}
\hline Activités & Langue maternelle & Langue 2 & Langue 3 \\
\hline Écouter la radio/regarder la télé & & & \\
\hline Lire pour les loisirs & & & \\
\hline Lire pour le travail & & & \\
\hline Lire sur internet & & & \\
\hline Écrire à des amis & & & \\
\hline Écrire des articles/essais & & & \\
\hline Autres & & & \\
\hline
\end{tabular}


I2. Quel est l'usage de ces langues par jour pour les communications suivantes (/heure)?

\begin{tabular}{|l|l|l|l|l|}
\hline Langue & Famille & Amis & Camarades & Collègues \\
\hline & & & & \\
\hline & & & & \\
\hline & & & & \\
\hline & & & & \\
\hline
\end{tabular}

I3. Avez-vous déjà vécu dans un pays étranger?

- Où?

- Combien de temps?

I4. Estimez votre capacité à apprendre des langues étrangères (I très bas, 2 bas, 3 moyen, 4 neutre, 5 bon, 6 très bon et 7 excellent):

$$
-\mathrm{I}-2-3-4-5-6-7
$$

I5. Si vous avez passé un diplôme dans les langues étrangères, indiquez le nom, le niveau et le résultat: 